\title{
DIVERGENT SERIES AND SINGULAR POINTS OF ORDINARY DIFFERENTIAL EQUATIONS*
}

\author{
BY \\ GEORGE.D. BIRKHOFF AND FREDERIC R. BAMFORTH $†$
}

INTRODUCTION

The analytic form of the solutions of the system of differential equations

$$
\frac{d x_{1}}{X_{1}\left(x_{1}, \cdots, x_{n}\right)}=\cdots=\frac{d x_{n}}{X_{n}\left(x_{1}, \cdots, x_{n}\right)}
$$

in a neighborhood of the point $x_{1}=\cdots=x_{n}=0$, in which all the functions $X_{i}$ are supposed to be analytic, $X_{i}(0, \cdots, 0)$ being zero for $i=1,2, \cdots$, $n$, has been the subject of much study. This is justified by the applications which can be made of this form to various theories in analysis and in dynamics. Dulac $\ddagger$ has simplified the problem in many cases by reducing the equations (1) to simple reduced forms of which the integration can be made without difficulty. The integration of these reduced equations then furnishes the solution of the system (1) either in terms of a parameter or in the form of a system of integrals.

Let $m_{i}$ be the roots of the so-called characteristic equation which, when written in determinant form, is

$$
\left|\frac{\partial X_{i}}{\partial x_{j}}-m \delta_{i j}\right|_{x_{1}=\cdots=x_{n}=0}=0,
$$

where $\delta_{i j}=0$ if $i \neq j$ and 1 if $i=j$, and let $L_{i}$ represent the linear terms of $X_{i}$, $i=1,2, \cdots, n$, when these functions are expanded according to powers of $x_{1}, \cdots, x_{n}$. Three conditions which have played an important rôle in the study of the system (1) may now be written as follows: (i) if the numbers $m_{i}$ are represented on a complex plane, there exists a straight line passing through the origin which is such that all the points $m_{i}$ are on the same side of it; (ii) there exists a linear change of variables $x_{i}=x_{i}\left(y_{1}, \cdots, y_{n}\right)$ such that the system of differential equations

$$
\frac{d x_{i}}{d \tau}=L_{i}
$$

* Presented to the Society, March 29, 1929; received by the editors May 15, 1929.

$\dagger$ National Research Fellow.

\$ Two papers to which reference will be made in the text are the following: $I$ : $H$. Dulac, Bulletin de la Société Mathématique de France, vol. 40 (1912), p. 324 et seq.; II: G. D. Birkhoff, Berlin Sitzungsberichte, 1929, pp. 171-183. 
is equivalent to the system

$$
\frac{d y_{i}}{d \tau}=m_{i} y_{i}
$$

(iii) there exists among the numbers $m_{i}$ no relationship of the form

$$
m_{i}=p_{1} m_{1}+\cdots+p_{n} m_{n},
$$

$p_{j}$ being positive integers whose sum is greater than 1 . We shall refer to these conditions frequently.

On introducing a parameter $t$ it is seen that the system (1) can be reduced to the system

$$
\frac{d x_{i}}{d t}=X_{i}\left(x_{1}, \cdots, x_{n}\right) \quad(i=1,2, \cdots, n) .
$$

The following lemma can be readily proved* by the aid of a theorem of Poincaré's and is already in the literature:

Lemma A. When the conditions (i), (ii) and (iii) are fulfilled, the system of differential equations (3) is equivalent in a neighborhood of $x_{1}=\cdots=x_{n}=0$ to the system

$$
\frac{d z_{i}}{d t}=m_{i} z_{i} \quad(i=1,2, \cdots, n),
$$

by means of a one-to-one analytic transformation of the form

$$
z_{i}=\phi_{i}\left(x_{1}, \cdots, x_{n}\right) \quad(i=1,2, \cdots, n),
$$

which leaves the point $x_{1}=x_{2}=\cdots=x_{n}=0$ invariant.

That this transformation is analytic is due to the fact that the formal power series solutions of the partial differential equations

$$
\frac{\partial \phi_{i}}{\partial x_{1}} X_{1}+\cdots+\frac{\partial \phi_{i}}{\partial x_{n}} X_{n}=m_{i} \phi_{i} \quad(i=1, \cdots, n)
$$

converge for $\left|x_{i}\right|<r, r>0$. But when the $m_{i}$ satisfy the conditions (ii) and (iii) and not the condition (i) in the case of real variables, the power series solutions of the equations (6) may diverge. Nevertheless, as has been conjectured, $\dagger$ even though these series diverge there may exist transformations playing the same rôle in which the functions $\phi_{i}$ are of class $C^{\infty}$ in a neighborhood of the origin and are analytic at every point of this neighborhood except 
possibly those which lie on certain manifolds of dimensions less than $n$. The existence of such a transformation has been proved* for the outstanding case $n=2$ in which $m_{1}$ is greater than zero, and $m_{2}$ is less than zero. In the present paper an analogous transformation is obtained for the case $n=3$ in which the $m_{i}$ are real and not all of one sign. It seems likely that the remaining important case $n=3$ not yet treated, in which $m_{2}$ and $m_{3}$ are conjugate imaginaries with real part of opposite sign to $m_{1}$, will offer essentially no new difficulties.

The case $n=3$ here treated differs essentially from the case $n=2$ in that for the former it is necessary to define two so-called invariant surfaces. The cases for $n>3$ seem to offer further difficulties. These will be discussed more fully in another paper.

Since in Section I we shall make use of another lemma which is already in the literature, $\uparrow$ we shall quote it here in a restricted form as

LEMMA B. When the right hand sides of the differential equations (3) have the form $m_{i} x_{i}+X_{2 i}, i=1,2, \cdots, n$, where $X_{2 i}$ have no terms of degree less than two, $m_{1}, \cdots, m_{p}$ are greater than zero, $m_{p+1}, \cdots, m_{n}$ are less than zero, and the condition (iii) is satisfied, there exists a transformation of the form

$$
\begin{array}{lrl}
y_{i}=x_{i}+f_{i}\left(x_{p+1}, \cdots, x_{n}\right) & (i=1, \cdots, p), \\
y_{j}=x_{j} & (j=p+1, \cdots, n),
\end{array}
$$

in which the functions $f_{i}$ are analytic in their variables in a neighborhood of $x_{p+1}=\cdots=x_{n}=0$, which reduces the differential equations (3) to ones of the same type in $y_{k}$ with the additional property that the function on the right hand side of any ith equation vanishes when $y_{1}=\cdots=y_{p}=0$.

\section{Preliminary analytic normalization}

Consider the system of differential equations

$$
\frac{d x_{i}}{d t}=X_{i}\left(x_{1}, x_{2}, x_{3}\right) \quad(i=1,2,3),
$$

in which the $X_{i}$ are real power series in the $x_{j}$, have no constant terms, and converge for $\left|x_{i}\right| \leqq r_{1}$ where $r_{1}>0$. The coefficients in the $X_{i}$ are constants and not functions of the parameter $t$. Let $m_{1}, m_{2}$ and $m_{3}$ be the roots of the determinant equation

* Loc. cit. II.

$$
\left|\frac{\partial X_{i}}{\partial x_{i}}-m \delta_{i j}\right|_{x_{1=x_{i}=x_{i}=0}}=0 .
$$

$\dagger$ I, p. 359 et seq. 
It will be assumed that $m_{1}$ and $m_{2}$ are greater than zero, $m_{3}$ is less than zero, and that the conditions (ii) and (iii) mentioned in the Introduction are satisfied.

The object of this paper is to show that the system of differential equations (7) is equivalent to the system

$$
\frac{d z_{i}^{*}}{d t}=m_{i} z_{i}^{*} \quad(i=1,2,3),
$$

by means of a one-to-one transformation of the type

$$
z_{i}^{*}=\phi_{i}\left(x_{1}, x_{2}, x_{3}\right)
$$

where the functions on the right involve the displayed arguments only, are zero at the origin, $x_{1}=x_{2}=x_{3}=0$, are continuous together with all their partial derivatives within a certain neighborhood of the origin, are analytic at any point of this neighborhood which does not lie on any one of a certain set of three surfaces which pass through the origin, and have certain further analyticity properties. If $m_{3}$ were positive instead of negative and the conditions (ii) and (iii) were still satisfied, then the transformation (9) might be chosen analytic in a neighborhood of the origin as Lemma A shows. However, when $m_{3}$ is negative, a transformation of the type just mentioned is the closest approach to an analytic transformation which we have discovered that will reduce the equations (7) to the equations (8). The equivalence of these two systems will only be shown to exist for a certain small neighborhood of the origin.

By an "invariant surface" will be meant a surface in the space of the dependent variables which is made up of integral curves of the differential equations (7) and contains the origin as an interior point; by an "invariant curve" will be meant an integral curve of (7) which passes through the origin. Now the classical theory of the system of differential equations (7) tells us that there exists an analytic invariant surface which contains two analytic invariant curves, and that there exists a third analytic invariant curve which does not lie in this surface. The first sequence of transformations which will be used will reduce the equations (7) to a special form in which these three analytic invariant curves are the three axes in the space of the dependent variables and the analytic invariant surface is one of the coordinate planes.

On account of the assumption that the condition (ii) is satisfied, we may assume that the right hand members of the differential equations (7) have the forms

$$
X_{i}=m_{i} x_{i}+X_{2 i}\left(x_{1}, x_{2}, x_{3}\right) \quad(i=1,2,3),
$$


in which the $X_{2 i}$ have no terms of less than the second degree in their arguments when they are expanded as power series about the origin.

Now let us examine the differential equations (7) whose right hand members have the forms given in (10) in the light of Lemmas A and B of the Introduction. Since we may perform the two analytic transformations which Lemma $B$ assures us exist, the first being obtained when we consider $m_{1}, m_{2}$ as the $m_{1}, \cdots, m_{p}$ of the lemma, and the second when we change $t$ to $-t$ and consider $m_{3}$ as the $m_{1}, \cdots, m_{p}$ of the lemma, we may assume that the $X_{2 i}$ of (10) have the property that

$$
X_{21}\left(0,0, x_{3}\right)=X_{22}\left(0,0, x_{3}\right)=X_{23}\left(x_{1}, x_{2}, 0\right)=0 .
$$

The performing of these transformations moves the third analytic invariant curve and the analytic invariant surface, which have been mentioned before, into the $x_{1}=x_{2}=0$ axis and the $x_{3}=0$ plane, respectively.

We wish now to move the two analytic invariant curves in the $x_{3}=0$ plane into the coördinate axes of that plane as well as make further simplifications. Since we wish to work in the $x_{3}=0$ plane, let us set $x_{3}=0$ which is seen to satisfy the third differential equation of (7) on account of the equations (11). The other two equations of (7) then take the form

$$
\frac{d x_{1}}{d t}=m_{1} x_{1}+X_{21}\left(x_{1}, x_{2}, 0\right), \frac{d x_{2}}{d t}=m_{2} x_{2}+X_{22}\left(x_{1}, x_{2}, 0\right) \text {. }
$$

In as much as these equations satisfy the hypotheses of Lemma $A$, and we may perform the transformation which that lemma says exists, we may assume that

$$
X_{21}\left(x_{1}, x_{2}, 0\right)=X_{22}\left(x_{1}, x_{2}, 0\right)=0
$$

which implies that the analytic invariant curves in the $x_{3}=0$ plane are the coördinate axes.

Since, on account of the equations (11), which are still true after the transformation just mentioned has been performed, $x_{1}=0, x_{2}=0$ satisfies the first two equations of (7), we may assume that $X_{23}\left(0,0, x_{3}\right)=0$, because if it were not so we could make use of an analytic transformation of the form

$$
y_{1}=x_{1}, y_{2}=x_{2}, y_{3}=x_{3}+f\left(x_{3}\right)
$$

whose existence is assured by Lemma A, to make $Y_{23}\left(0,0, y_{3}\right)=0$. 
As a result of this discussion we may state that without loss of generality, the differential equations (7) may be assumed to have the form

$$
\begin{aligned}
& \frac{d x_{1}}{d t}=x_{1}\left(m_{1}+x_{3} P_{1}\right)+x_{2} x_{3} Q_{1}, \\
& \frac{d x_{2}}{d t}=x_{2}\left(m_{2}+x_{3} P_{2}\right)+x_{1} x_{3} Q_{2}, \\
& \frac{d x_{3}}{d t}=x_{3}\left(m_{3}+x_{1} P_{3}+x_{2} P_{4}\right),
\end{aligned}
$$

where the $P_{i}$ and $Q_{i}$ are analytic functions of their arguments $x_{j}$ in a neighborhood of the origin, for $\left|x_{i}\right| \leqq r, r>0, i=1,2,3, Q_{1}$ is a function of $x_{2}$ and $x_{3}$ only, and $Q_{2}$ is a function of $x_{1}$ and $x_{3}$ only.

\section{CONVERgence PROPERTIES OF FORMAL SERIES}

Let us recall that the aim of the present paper is to find three functions $\phi_{i}\left(x_{1}, x_{2}, x_{3}\right)$ such that, when the $x_{j}$ are replaced by an arbitrary solution $x_{j}(t)$ of the differential equations $(12), z_{i}^{*}=\phi_{i}\left[x_{1}(t), x_{2}(t), x_{3}(t)\right]$ satisfy the differential equations (8). Since all three of these functions can be found in exactly the same way we shall confine our attention for the present to $\phi_{1}$. Evidently $\phi_{1}$ must be a solution of the partial differential equation

$$
\begin{aligned}
\frac{\partial \phi_{1}}{\partial x_{1}}\left[x_{1}\left(m_{1}+x_{3} P_{1}\right)+x_{2} x_{3} Q_{1}\right] & +\frac{\partial \phi_{1}}{\partial x_{2}}\left[x_{2}\left(m_{2}+x_{3} P_{2}\right)+x_{1} x_{3} Q_{2}\right] \\
& +\frac{\partial \phi_{1}}{\partial x_{3}}\left[x_{3}\left(m_{3}+x_{1} P_{3}+x_{2} P_{4}\right)\right]=m_{1} \phi_{1} .
\end{aligned}
$$

Equation (13) has a formal power series solution whose only linear term is $a x_{1}$, where $a$ is an arbitrary constant. This power series solution is unique if the coefficient of $x_{1}$ is chosen as 1 . Let us assume that this has been done. Furthermore, every other term has a factor $x_{1} x_{3}$ or $x_{2} x_{3}$ as is readily seen by alternately setting $x_{1}=x_{2}=0$ and $x_{3}=0$ in (13) and solving for $\phi_{1}$. Let this formal series be

$$
\phi_{1} \sim x_{1}+\cdots+\frac{a_{m n p}}{m ! n ! p !} x_{1}^{m} x_{2}^{n} x_{3}^{p}+\cdots,
$$

which obviously can be written in either of the forms

$$
\phi_{1} \sim \alpha_{0}\left(x_{1}, x_{2}\right)+\alpha_{1}\left(x_{1}, x_{2}\right) x_{3}+\cdots+\alpha_{p}\left(x_{1}, x_{2}\right) x_{3}^{p}+\cdots,
$$




$$
\phi_{1} \sim \alpha_{00}\left(x_{3}\right)+\alpha_{10}\left(x_{3}\right) x_{1}+\cdots+\alpha_{m n}\left(x_{3}\right) x_{1}{ }^{m} x_{2}{ }^{n}+\cdots .
$$

We shall now prove

LEMMA 1. The series $\alpha_{p}$ of (15) and the series $\alpha_{m n}$ of (16) all converge for $\left|x_{1}\right|,\left|x_{2}\right|<r$ and for $\left|x_{3}\right|<r$ respectively.

Substitute the series (15) into the equation (13) and equate the coefficients of like powers of $x_{3}$. Evidently $\alpha_{0}=x_{1}$, and $\alpha_{1}$ satisfies the equation

$$
\frac{\partial \alpha_{1}}{\partial x_{1}} x_{1} m_{1}+\frac{\partial \alpha_{1}}{\partial x_{2}} x_{2} m_{2}+\alpha_{1}\left(m_{3}-m_{1}+x_{1} P_{30}+x_{2} P_{40}\right)=\Phi_{1},
$$

where $P_{i 0}=P_{i}\left(x_{1}, x_{2}, 0\right)$ and $\Phi_{1}$ is linear in $\alpha_{0}$ with coefficients which are analytic in $x_{1}, x_{2}$ for $\left|x_{1}\right|,\left|x_{2}\right| \leqq r$, and are zero for $x_{1}=x_{2}=0$.

Set

$$
\alpha_{1}=\beta_{0}\left(x_{2}\right)+\cdots+\beta_{s}\left(x_{2}\right) x_{1}^{s}+\cdots,
$$

where the $\beta_{i}$ are formal power series in $x_{2}$. Then $\beta_{0}$ formally satisfies an equation of the form

$$
\frac{d \beta_{0}}{d x_{2}} x_{2} m_{2}+\beta_{0}\left(m_{3}-m_{1}+x_{2} P_{400}\right)=\Phi_{10},
$$

where $P_{400}$ and $\Phi_{10}$ are analytic in $x_{2}$ for $\left|x_{2}\right| \leqq r$. On account of the condition (iii) of the Introduction being satisfied, it readily follows that $\beta_{0}$ is analytic $\dagger$ in $x_{2}$ for $\left|x_{2}\right| \leqq r$. In a similar manner it can be shown that all the $\beta_{i}$ are analytic in $x_{2}$ for $\left|x_{2}\right| \leqq r$, and if $\alpha_{1}$ be arranged according to ascending powers of $x_{2}$ it can be shown in the same manner that the coefficients are analytic in $x_{1}$ for $\left|x_{1}\right| \leqq r$.

Now define

$$
B_{s}=\beta_{0}+\beta_{1} x_{1}+\cdots+\beta_{s-1} x_{1}^{s-1}
$$

and $\gamma_{1}=\alpha_{1}-B_{8}$. Evidently $\gamma_{1}$ has a factor $x_{1}^{8}$, and hence, writing $\gamma_{1}=x_{1}^{8} \xi_{1}$, we see that $\xi_{1}$ will satisfy an equation of the form

$$
\frac{\partial \xi_{1}}{\partial x_{1}} x_{1} m_{1}+\frac{\partial \xi_{1}}{\partial x_{2}} x_{2} m_{2}+\xi_{1}\left(m_{3}-m_{1}+s m_{1}+x_{1} P_{30}+x_{2} P_{40}\right)=\Phi_{11},
$$

where $\Phi_{11}$ is analytic in $x_{1}, x_{2}$ for $\left|x_{1}\right|,\left|x_{2}\right| \leqq r$. Let $s$ be large enough so that $m_{3}+m_{1}(s-1)$ is positive and define $m_{4}$ as equal to this quantity. Let $m$ be a number that is greater than zero and less than the smallest of $m_{1}, m_{2}$ and $m_{4}$. Then

\footnotetext{
† Cf. II, pp. 177-178.
}

$$
\left|p m_{1}+q m_{2}+m_{4}\right|>m(p+q+1)
$$


for any pair of positive integers $p, q$. Choose $M>0$ and such that $x_{1} P_{30}$ and $x_{2} P_{40}$ are dominated by

$$
\frac{M}{\left(1-\frac{x_{1}}{r}\right)\left(1-\frac{x_{2}}{r}\right)}-M
$$

and $\Phi_{11}$ is dominated by the first term of (19) only. Then the power series $\xi_{1}$ is dominated by the power series solution of

$$
\begin{gathered}
m\left(x_{1} \frac{\partial f}{\partial x_{1}}+x_{2} \frac{\partial f}{\partial x_{2}}\right)=f\left[-m+\frac{M}{\left(1-\frac{x_{1}}{r}\right)\left(1-\frac{x_{2}}{r}\right)}-M\right] \\
+\frac{M}{\left(1-\frac{x_{1}}{r}\right)\left(1-\frac{x_{2}}{r}\right)} .
\end{gathered}
$$

On account of the symmetry in (20) with respect to $x_{1}$ and $x_{2}$ the power series solution of (20) converges for $\left|x_{1}\right|,\left|x_{2}\right|<R$, where $R$ is the radius of convergence of the power series solution of the differential equation

$$
m x_{1} \frac{d F}{d x_{1}}=F\left[-m+\frac{M}{\left(1-\frac{x_{1}}{r}\right)^{2}}-M\right]+\frac{M}{\left(1-\frac{x_{1}}{r}\right)^{2}} .
$$

From this it follows that $R=r$. Hence $\alpha_{1}$ is analytic in $x_{1}, x_{2}$ for $\left|x_{1}\right|,\left|x_{2}\right|<r$.

The proof of the lemma for the other $\alpha_{i}$ follows readily by induction since every $\alpha_{i}$ satisfies an equation of the form (17) where $\alpha_{1}$ is replaced by $\alpha_{i}, m_{3}$ by $i m_{3}$, and $\Phi_{1}$ by $\Phi_{i}$, where $\Phi_{i}$ is a polynomial in $\alpha_{0}, \cdots, \alpha_{i-1}$, with coefficients which are analytic in $x_{1}, x_{2}$ for $\left|x_{1}\right|,\left|x_{2}\right| \leqq r$.

The $\alpha_{m n}$ can be proved to be analytic in the same way that the $\beta_{i}$ of (18) are proved analytic. This completes the proof of Lemma 1.

Similarly, we may prove that when the series corresponding to $\phi_{2}$ and $\phi_{3}$ are arranged as power series in $x_{3}$ the coefficients are analytic in $x_{1}, x_{2}$ for $\left|x_{1}\right|,\left|x_{2}\right|<r$, and when arranged as power series in $x_{1}, x_{2}$ the coefficients are analytic in $x_{3}$ for $\left|x_{3}\right|<r$. The only linear term in $\phi_{2}$ is the one involving $x_{2}$, and every other term contains as a factor $x_{1} x_{3}$ or $x_{2} x_{3}$. The coefficient of the linear term is arbitrary and when it is chosen as 1 the series for $\phi_{2}$ is unique. Let us suppose that this choice has been made. The series for $\phi_{3}$ has properties similar to those of $\phi_{1}$ and $\phi_{2}$ with the additional one that $x_{3}$ is a factor of every term in the series. We shall choose the coefficient of 
the linear term as 1 and this choice uniquely determines all the others.

If the formal series for $\phi_{i}, i=1,2$, or 3 , should converge for $\left|x_{i}\right|<r_{0}$, $r_{0}>0, i=1,2,3$, it can evidently be used to reduce the $i$ th differential equation of (12) immediately to the normal form $d z_{i}^{*} / d t=m_{i} z_{i}^{*}$.

Even though the series for the $\phi_{i}$ should diverge, it will be found that they can be "fitted" by functions of class $C^{\infty}$ in a manner described in

LEMMA 2. Let the formal series

$$
S\left(x_{1}, x_{2}, x_{3}\right)=\sum_{m, n, p=0}^{\infty} \frac{a_{m n p}}{m ! n ! p !} x_{1}^{m} x_{2}^{n} x_{3}^{p},
$$

in which all the $a_{m n p}$ are real, have the property that when it is arranged as a power series in $x_{3}$, i.e.,

$$
S=\alpha_{0}\left(x_{1}, x_{2}\right)+\alpha_{1}\left(x_{1}, x_{2}\right) x_{3}+\cdots+\alpha_{p}\left(x_{1}, x_{2}\right) x_{3}{ }^{p} / p !+\cdots,
$$

the $\alpha_{p}$ are analytic in the complex variables $x_{1}, x_{2}$ for $\left|x_{1}\right|,\left|x_{2}\right| \leqq r, r>0$, and when it is arranged as a power series in $x_{1}, x_{2}$, i.e.,

$$
S=\alpha_{00}\left(x_{3}\right)+\alpha_{10}\left(x_{3}\right) x_{1}+\cdots+\alpha_{m n}\left(x_{3}\right) x_{1}{ }^{m} x_{2}{ }^{n} /(m ! n !)+\cdots,
$$

the $\alpha_{m n}$ are analytic in the complex variable $x_{3}$ for $\left|x_{3}\right| \leqq r$. Then there exists a real function $F\left(x_{1}, x_{2}, x_{3}\right)$ of the real variables $x_{1}, x_{2}, x_{3}$ which is continuous together with all its partial derivatives for $\left|x_{1}\right|,\left|x_{2}\right|,\left|x_{3}\right| \leqq r$, is analytic for $x_{1} \neq 0, x_{2} \neq 0, x_{3} \neq 0$, satisfies the equations

$$
\left.\frac{\partial^{m+n} F}{\partial x_{1}^{m} \partial x_{2}^{n}}\right|_{x_{1}=x_{2}=0}=\alpha_{m n}\left(x_{3}\right),\left.\quad \frac{\partial^{p} F}{\partial x_{3}^{p}}\right|_{x_{s}=0}=\alpha_{p}\left(x_{1}, x_{2}\right)(m, n, p=0,1, \cdots),
$$

and has the property that it and all its partial derivatives are analytic in $x_{i}$ and $x_{i}$ for $\left|x_{i}\right|,\left|x_{i}\right| \leqq r, x_{i} \neq 0, x_{j} \neq 0, x_{k}=0, i \neq j, i \neq k, j \neq k$, and are analytic in $x_{i}$ for $\left|x_{i}\right| \leqq r, x_{i}=x_{k}=0, i, j, k=1,2,3$.

Consider the series $\dagger$

$$
F\left(x_{1}, x_{2}, x_{3}\right)=\sum_{m, n, p=0}^{\infty} \frac{a_{m n p}}{m ! n ! p !} x_{1}^{m} x_{2}^{n} x_{3}^{p}\left(1-e^{\left.-1 / B_{m n p}\right)},\right.
$$

where $i=1$ if $m \geqq n, i=2$ if $m<n, b_{m n p}=1+\left|a_{m n p}\right|, B_{m n p}=b_{m n p} x_{3}{ }^{2} x_{i}{ }^{2}$. At first we shall consider this series only in the six-dimensional complex region defined by

$$
\left|x_{i}\right| \leqq r, \quad\left|\arg x_{i}\right| \leqq \theta, \quad 0<\theta<\pi / 8 \quad(j=1,2,3) .
$$

In this region $\left|\arg x_{3}^{2} x_{i}^{2}\right|$ does not exceed $\pi / 2$, so that the exponential † Cf. II, p. 173. 
terms $e^{-1 / B_{m n p}}$ in the series have exponents $-1 / B_{m n p}$ with negative real parts, and so are less than one in absolute value.

Moreover, we find for $\left|B_{m n p}\right| \geqq 1$,

$$
\begin{aligned}
\left|a_{m n p}\left(1-e^{-1 / B_{m n p}}\right)\right| & =\left|a_{m n p}\right| \cdot\left|\frac{1}{B_{m n p}}-\frac{1}{2 ! B_{m n p}^{2}}+\cdots\right| \\
& \leqq\left|a_{m n p}\right| \cdot(e-1) /\left|B_{m n p}\right| \leqq 2 /\left[\left|x_{3}\right|^{2}\left|x_{i}\right|^{2}\right] .
\end{aligned}
$$

But if, on the contrary, $\left|B_{m n p}\right|<1$, then, by the definition of $B_{m n p},\left|a_{m n p}\right| \leqq$ $1 /\left[\left|x_{3}\right|^{2}\left|x_{i}\right|^{2}\right]$. Since the quantity in the parentheses in the preceding inequality is obviously less than two in absolute value, we conclude that the inequality holds in this case too and so without restriction on $B_{m n p}$. Thus the given series is dominated by the series

where

$$
\sum_{m, n, p=0}^{\infty} \frac{2}{m ! n ! p !}\left|x_{1}\right|^{m_{1}}\left|x_{2}\right|^{n_{1}}\left|x_{3}\right|^{p-2}
$$

$$
\begin{aligned}
& m_{1}=m-2 \text { if } m \geqq n \text { and } m \text { if } m<n, \\
& n_{1}=n-2 \text { if } m<n \text { and } n \text { if } m \geqq n .
\end{aligned}
$$

But it follows from the hypotheses concerning the $\alpha_{p}$ that that part of the series (24) for which $p \leqq 3$ converges uniformly in the region (25); and it follows from the hypotheses concerning the $\alpha_{m n}$ that that part of the series (24) which gives rise to a negative $m_{1}$ or to a negative $n_{1}$ converges uniformly in the same region. Hence the series (24) converges uniformly in the region (25), and an examination of this series will readily show that it is analytic except for $x_{1}=0, x_{2}=0$, or for $x_{3}=0$, and that

$$
F\left(x_{1}, x_{2}, 0\right)=\alpha_{0}, \quad F\left(0,0, x_{3}\right)=\alpha_{00} .
$$

In a similar manner, all the series obtained from $F$ by partial differentiation can be shown to have the same properties of analyticity and continuity as has $F$ in the region defined by (25). This is on account of the fact that the $\alpha_{p}$ of (21) and the $\alpha_{m n}$ of (22) are analytic for $\left|x_{1}\right|,\left|x_{2}\right|,\left|x_{3}\right| \leqq r$ and that for a finite number of differentiations of (24) only a finite number of negative powers of $x_{1}$ and of $x_{2}$ are introduced into the dominating series which correspond to these derivative series in the same way that the series (26) does to $F$. We shall consider only $\partial F / \partial x_{3}$,

$$
\begin{aligned}
\frac{\partial F}{\partial x_{3}}=\sum_{m, n, p-1=0}^{\infty} \frac{a_{m n p}}{m ! n !(p-1) !} x_{1}^{m} x_{2}^{n} x_{3}^{p-1}\left(1-e^{\left.-1 / B_{m n p}\right)}\right. & \\
& \quad+\sum_{m, n, p=0}^{\infty} \frac{a_{m n p}}{m ! n ! p !} x_{1}^{m} x_{2}{ }^{n} x_{3}{ }^{p}\left(\frac{-2 e^{-1 / B_{m n p}}}{B_{m n p} x_{3}}\right) .
\end{aligned}
$$


The first of these series is evidently dominated by

$$
\sum_{m, n, p-1=0}^{\infty} \frac{2\left|x_{1}\right|^{m_{1}}\left|x_{2}\right|^{n_{1}}\left|x_{3}\right|^{p-3}}{m ! n !(p-1) !}
$$

and the second by

$$
\sum_{m, n, p=0}^{\infty} \frac{2\left|x_{1}\right|^{m_{1}}\left|x_{2}\right|^{n_{1}}\left|x_{3}\right|^{p-3}}{m ! n ! p !} .
$$

From the remarks made above concerning $\alpha_{p}$ and $\alpha_{m n}$, etc., it readily follows that $\partial F / \partial x_{3}$ has the same properties of continuity and analyticity as has $F$ itself. Furthermore,

$$
\left.\frac{\partial F}{\partial x_{3}}\right|_{x_{2}=0}=\alpha_{1} .
$$

$F\left(x_{1}, x_{2}, x_{3}\right)$ is thus a real function in the octant $x_{1} \geqq 0, x_{2} \geqq 0, x_{3} \geqq 0$, continuous together with all its partial derivatives in this octant, analytic for $x_{1}>0, x_{2}>0, x_{3}>0$, and satisfies the equations (23). Similarly $F$ is seen to have the same properties in each of the other octants and the eight functions thus defined evidently unite to form a function $F\left(x_{1}, x_{2}, x_{3}\right)$ defined by the series (24) and having the properties stated in the lemma down to and including those relative to the equations (23). To prove the last statements made in the lemma concerning the function $F$ and its partial derivaatives we need merely use dominating series in a manner wholly analogous to that in which they were used above.

\section{Final analytic NoRmalization}

The problem in hand is more readily discussed if the functions $Q_{1}$ and $Q_{2}$ appearing in the differential equations (12) have the factors $x_{2} x_{3}$ and $x_{1} x_{3}$, respectively. We shall now show that the assumption that such is the case can be made without the introduction of any further hypotheses than have already been made. To this end let us consider the transformation

$$
y_{i}=s_{i}\left(x_{1}, x_{2}, x_{3}\right) \quad(i=1,2,3),
$$

where the series $s_{i}\left(x_{1}, x_{2}, x_{3}\right)$ consist of all the terms of the formal series for $\phi_{i}, i=1,2,3$, respectively, involving $x_{1}{ }^{m} x_{2}{ }^{n}, m+n=0,1, \cdots, \lambda+1$, all the terms involving $x_{3}{ }^{p}, p=0,1, \cdots, \lambda+1$, and no others. For the present $\lambda$ will be considered as any integer greater than 1 but will be determined in a later section as depending on the $m_{i}$. The properties of convergence of the coefficients of the formal series for the $\phi_{i}$ when these series are arranged according to powers of $x_{3}$ or according to powers of $x_{1}$ and $x_{2}$ imply that the series $s_{i}$ converge for $\left|x_{j}\right|<r$. 
From the definition of the $s_{i}$ it follows that

$$
\begin{aligned}
\frac{\partial s_{i}}{\partial x_{1}}\left[x_{1}\left(m_{1}+x_{3} P_{1}\right)+x_{2} x_{3} Q_{1}\right]+\frac{\partial s_{i}}{\partial x_{2}}\left[x _ { 2 } \left(m_{2}\right.\right. & \left.\left.+x_{3} P_{2}\right)+x_{1} x_{2} Q_{2}\right] \\
& +\frac{\partial s_{i}}{\partial x_{3}} x_{3}\left(m_{3}+x_{1} P_{3}+x_{2} P_{4}\right)-m_{i} s_{i}
\end{aligned}
$$

define functions $R_{i}\left(x_{1}, x_{2}, x_{3}\right)$ which have no terms of degree less than $\lambda+2$ in $x_{3}$ and less than $\lambda+2$ in $x_{1}$ and $x_{2}$ together. Since the only linear term in $s_{i}$ is $x_{i}, i=1,2,3$, respectively, when we solve (28) for $x_{i}=t_{i}\left(y_{1}, y_{2}, y_{3}\right)$ the only linear term in $t_{i}$ is $y_{i}, i=1,2,3$, respectively. Hence the $R_{i}$ when expressed as functions of $y_{j}$ have no terms of degree less than $\lambda+2$ in $y_{3}$ and less than $\lambda+2$ in $y_{1}$ and $y_{2}$ together. Thus, when $x_{i}$ are replaced by an arbitrary solution $x_{i}(t)$ of (12), the corresponding $y_{i}$ satisfy the system of differential equations

$$
\frac{d y_{i}}{d t}=m_{i} y_{i}+R_{i} \quad(i=1,2,3),
$$

where the arguments of the $R_{i}$ are the $y_{j}$. But every term in the formal series for $\phi_{1}$, except the linear term, contains $x_{1} x_{3}$ or $x_{2} x_{3}$ as a factor, and corresponding statements are true concerning the formal series for the $\phi_{2}$ and $\phi_{3}$. This implies that when $x_{3}=0$, then $y_{3}=0, x_{1}=y_{1}$ and $x_{2}=y_{2}$, and when $x_{1}=x_{2}=0$, then $y_{1}=y_{2}=0$ and $x_{3}=y_{3}$. Thus the transformation (28) will preserve the form of the differential equations (12) and hence the form of the system (29) is the same as that of the system (12) when the $x$ are replaced by the $y_{i}, i=1,2,3$. Furthermore, on account of the properties of the $R_{i}$ we see that the new $Q_{1}$ will have $y_{2}{ }^{\lambda} y_{3}{ }^{\lambda}$ as a factor and the new $Q_{2}$ will have ${ }_{1}{ }^{\lambda} y_{3}{ }^{\lambda}$ as a factor. Hence we are not losing in generality when we assume that the differential equations under discussion have the form

$$
\begin{aligned}
& \frac{d x_{1}}{d t}=x_{1}\left(m_{1}+x_{3} P_{1}\right)+x_{2}^{2} x_{3}^{2} Q_{1}, \\
& \frac{d x_{2}}{d t}=x_{2}\left(m_{2}+x_{3} P_{2}\right)+x_{1}^{2} x_{3}^{2} Q_{2}, \\
& \frac{d x_{3}}{d t}=x_{3}\left(m_{3}+x_{1} P_{3}+x_{2} P_{4}\right),
\end{aligned}
$$

where the $P_{i}$ and $Q_{i}$ are analytic in $x_{j}$ for $\left|x_{j}\right| \leqq r, r>0, j=1,2,3, Q_{1}$ is a function of $x_{2}$ and $x_{3}$ only, and $Q_{2}$ is a function of $x_{1}$ and $x_{3}$ only. Furthermore, every $P_{i}$ has $x_{3}{ }^{\lambda+1}$ as a factor, and when expanded as a power series in 
$x_{1}$ and $x_{2}$ contains no term of degree less than $\lambda+1$ in $x_{1}$ and $x_{2}$ together; $Q_{1}$ contains $x_{2}{ }^{\lambda} x_{3}{ }^{\lambda}$ as a factor, and $Q_{2}$ contains $x_{1}{ }^{\lambda} x_{3}{ }^{\lambda}$ as a factor. These facts will be used in the next section. The partial differential equation (13) and the corresponding ones in $\phi_{2}$ and $\phi_{3}$ will now be replaced by the system

$$
\begin{aligned}
\frac{\partial \phi_{i}}{\partial x_{1}}\left[x _ { 1 } \left(m_{1}+\right.\right. & \left.\left.x_{3} P_{1}\right)+x_{2}^{2} x_{3}^{2} Q_{1}\right]+\frac{\partial \phi_{i}}{\partial x_{2}}\left[x_{2}\left(m_{2}+x_{3} P_{2}\right)+x_{1}^{2} x_{3}^{2} Q_{2}\right] \\
+ & \frac{\partial \phi_{i}}{\partial x_{3}}\left[x_{3}\left(m_{3}+x_{1} P_{3}+x_{2} P_{4}\right)\right]=m_{i} \phi_{i} \quad(i=1,2,3) .
\end{aligned}
$$

\section{CONSTRUCTION OF AUXILIARY INVARIANT SURFACES}

It happens that if $Q_{1}=Q_{2}=0$ we can fairly readily obtain solutions of this system (31) of class $C^{\infty}$ in a neighborhood of the origin, and thus solve our problem. Hence we shall try to find a transformation of the form

$$
x_{i}=x_{i}\left(y_{1}, y_{2}, y_{3}\right)
$$$$
(i=1,2,3) \text {, }
$$

which will reduce this system of partial differential equations to an equivalent system in which the coefficient of $\partial \phi_{i} / \partial y_{j}$ has $y_{j}$ as a factor, $i, j=1,2,3$. Evidently we may choose $x_{3}=y_{3}$ as the last equation in this transformation. Now if the system of partial differential equations when expressed in terms of the $y_{j}$ have this additional property, the transformation (32) will take the integral curves of the system of differential equations (30) into the integral curves of a system of differential equations of the form

$$
\frac{d y_{i}}{d t}=y_{i}\left(m_{i}+T_{i}\right) \quad(i=1,2,3) .
$$

Hence our search for such a transformation (32) is connected with the search for two invariant surfaces of the system of differential equations (30) other than the known one $x_{3}=0$. In this connection we shall prove

TheORem 1. There exists a function $f_{1}\left(x_{2}, x_{3}\right)$ which is of class $C^{\infty}$ in a neighborhood of $x_{2}=x_{3}=0$, is analytic in $x_{2}, x_{3}$ for $x_{2} \neq 0, x_{3} \neq 0$, is, together with all its partial derivatives, analytic in either variable when the other variable is zero, and is such that

$$
x_{1}=x_{2}{ }^{2} x_{3}{ }^{2} f_{1}\left(x_{2}, x_{3}\right)
$$

is an invariant surface. There also exists another function $g_{1}\left(x_{1}, x_{3}\right)$ with properties analogous to those of $f_{1}\left(x_{2}, x_{3}\right)$ such that

$$
x_{2}=x_{1}^{2} x_{3}^{2} g_{1}\left(x_{1}, x_{3}\right)
$$

is another invariant surface. 
Since the method to be used for the determination of one of these invariant surfaces can be used for the determination of the other, we shall confine our attention to that surface which will be transformed into the $y_{1}=0$ plane. To this end consider the transformation

$$
y_{1}=x_{1}-x_{2}^{2} x_{3}^{2} f_{1}\left(x_{2}, x_{3}\right), \quad y_{2}=x_{2}, \quad y_{3}=x_{3},
$$

where the function $f_{1}$ is to be so determined that when the $x_{i}$ are replaced by an arbitrary solution $x_{i}(t)$ of (30) the $y_{1}(t)$ so defined will satisfy a differential equation of the form

$$
\frac{d y_{1}}{d t}=y_{1}\left(m_{1}+T_{1}\right),
$$

in which $T_{1}$ is a function of the $y_{i}$ of class $C^{\infty}$ and $T_{1}(0,0,0)=0$. On differentiating the first equation of (33) with respect to $t$, replacing the derivatives $d x_{i} / d t$ by their values given in the equations (30) and $x_{1}$ by its value given in (33), and noting that $y_{1}=0$ is to be a solution of the resulting differential equation on account of the required form (34), we obtain the result that $f_{1}\left(x_{2}, x_{3}\right)$ is a solution of the partial differential equation

$$
\begin{gathered}
\frac{\partial f_{1}}{\partial x_{2}} x_{2}\left(m_{2}+x_{3} R_{1}\right)+\frac{\partial f_{1}}{\partial x_{3}} x_{3}\left(m_{3}+x_{2} R_{2}\right) \\
=f_{1}\left(m_{4}-2 x_{2} R_{2}+x_{3} R_{3}\right)+Q_{1}
\end{gathered}
$$

in which

$$
\begin{array}{ll}
m_{4}=m_{1}-2 m_{2}-2 m_{3}, & R_{1}=P_{2}+x_{2}^{3} x_{3}{ }^{5} f_{1}^{2} Q_{2}, \\
R_{2}=x_{2} x_{3}{ }^{2} f_{1} P_{3}+P_{4}, & R_{3}=P_{1}-2 R_{1},
\end{array}
$$

where the $P_{i}$ and the $Q_{2}$ are the same functions of $x_{2}^{2} x_{3}^{2} f_{1}, x_{2}, x_{3}$ as they were of $x_{1}, x_{2}, x_{3}$ in (30). From the fact that every $P_{i}$ has $x_{3}{ }^{\lambda+1}$ as a factor and when expanded as a power series in $x_{1}$ and $x_{2}$ contains no term of degree less than $\lambda+1$ in $x_{1}$ and $x_{2}$ together, and from the form of the $R_{i}$ it follows that every $R_{i}$ has $x_{2}{ }^{\lambda+1} x_{3}{ }^{\lambda+1}$ as a factor. If $f_{1}\left(x_{2}, x_{3}\right)$ is any solution of the equation (35) which is of class $C^{\infty}$ in a neighborhood of the origin, the function $y_{1}$ so defined by the first equation in (33) will satisfy an equation of the form (34) when the $x_{i}$ are replaced by an arbitrary solution $x_{i}(t)$ of the differential equations (30). This fact is an immediate consequence of the manner in which the partial differential equation (35) has been derived.

We shall now prove 
Lemma 3. There exists a solution $f_{1}\left(x_{2}, x_{3}\right)$ of the partial differential equation (35) which is of class $C^{\infty}$ in a neighborhood of the origin $x_{2}=x_{3}=0$ and is analytic at any interior point of this neighborhood for which $x_{2} \neq 0, x_{3} \neq 0$. Furthermore, this solution and all its partial derivatives are analytic in either variable in a neighborhood of $x_{i}=0, i=2$ or 3 as the case may be, when the other variable is zero.

From what has gone before, Theorem 1 will be obvious when Lemma 3 has been proved.

The first thing to be observed is that there exists a unique formal power series solution of the partial differential equation (35) which may be written in any one of the three forms

$$
\begin{aligned}
f_{1} & \sim a_{00}+\cdots+\frac{a_{m n}}{m ! n !} x_{2}^{m} x_{3}^{n}+\cdots \\
& =\alpha_{0}\left(x_{2}\right)+\cdots+\alpha_{m}\left(x_{2}\right) x_{3}{ }^{m}+\cdots \\
& =\beta_{0}\left(x_{3}\right)+\cdots+\beta_{n}\left(x_{3}\right) x_{2}{ }^{n}+\cdots
\end{aligned}
$$

Since every $R_{i}$ and $Q_{1}$ has $x_{2}^{\lambda} x_{3}^{\lambda}$ as a factor, this formal series for $f_{1}$ also has $x_{2}{ }^{\lambda} x_{3}{ }^{\lambda}$ as a factor. If this series does not diverge for every pair of values $x_{2} \neq 0, x_{3} \neq 0$, our lemma is obviously proved, so that we shall confine our attention in the following discussion to the contrary case. By making use of the methods employed in the proof of Lemma 1 it can be shown that all the $\alpha_{m}\left(x_{2}\right)$ are analytic in $x_{2}$ for $\left|x_{2}\right|<r$ and that all the $\beta_{n}\left(x_{3}\right)$ are analytic in $x_{3}$ for $\left|x_{3}\right|<r$. This follows from the fact that wherever $f_{1}$ appears in $P_{i}$ or in $Q_{2}$ it is multiplied by $x_{2}^{2} x_{3}^{2}$. Now define a function $g\left(x_{2}, x_{3}\right)$ which corresponds to the formal series for $f_{1}$ in the same way that the function $F$ corresponds to the series $S$ in Lemma 2, it being supposed for this application of this lemma that no $x_{1}$ appears in the series $S$ and that that part of the function $F$ which is independent of $x_{2}$ is replaced by the corresponding analytic series which is a part of $S$. On making the transformation $f_{1}=f+g$ we see that $f_{1}$ satisfies the differential equation (35) if and only if $f$ is a solution of the partial differential equation

$$
\frac{\partial f}{\partial x_{2}} x_{2}\left(m_{2}+x_{3} S_{1}\right)+\frac{\partial f}{\partial x_{3}} x_{3}\left(m_{3}+x_{2} S_{2}\right)=f\left(m_{4}+S_{3}\right)+S_{4},
$$

in which the $S_{i}$ are well defined functions of $f, x_{2}, x_{3}, S_{3}(0,0,0)$ being zero and $S_{4}$ being independent of $f$. These functions are given explicitly by the following equations (38) and have the properties that they are of class $C^{\infty}$ in a neighborhood of $f=x_{2}=x_{3}=0$, are analytic in $f$ in a neighborhood of $f=0$ for $x_{2}$ and $x_{3}$ in a neighborhood of $x_{2}=x_{3}=0$, and are analytic in $x_{2}, x_{3}$ in a 
neighborhood of $x_{2}=x_{3}=0$ for $x_{2} \neq 0, x_{3} \neq 0$ and $f$ in a neighborhood of $f=0$. All these properties are immediately implied by the equations

$$
\begin{aligned}
S_{1}\left(f, x_{2}, x_{3}\right)= & R_{1}\left(f+g, x_{2}, x_{3}\right), S_{2}\left(f, x_{2}, x_{3}\right)=R_{2}\left(f+g, x_{2}, x_{3}\right), \\
S_{3}\left(f, x_{2}, x_{3}\right)= & -2 x_{2} R_{2}+x_{3} R_{3}-\frac{1}{f}\left\{\frac{\partial g}{\partial x_{2}} x_{2} x_{3}\left(R_{1}-R_{10}\right)\right. \\
& +\frac{\partial g}{\partial x_{3}} x_{2} x_{3}\left(R_{2}-R_{20}\right)-g\left[-2 x_{2}\left(R_{2}-R_{20}\right)\right. \\
& \left.\left.+x_{3}\left(R_{3}-R_{30}\right)\right]\right\}, \\
S_{4}\left(f, x_{2}, x_{3}\right)= & Q_{1}+g\left(m_{4}-2 x_{2} R_{20}+x_{3} R_{30}\right)-\frac{\partial g}{\partial x_{2}} x_{2}\left(m_{2}+x_{3} R_{10}\right) \\
& -\frac{\partial g}{\partial x_{3}} x_{3}\left(m_{3}+x_{2} R_{20}\right),
\end{aligned}
$$

where the arguments of the $R_{i}$ are $f+g, x_{2}, x_{3}$ and $R_{i 0}=R_{i}\left(0+g, x_{2}, x_{3}\right)$. Evidently every $S_{i}$ has $x_{2}{ }^{\lambda} x_{3}{ }^{\lambda}$ as a factor. This fact will be made use of later on. From the definition of $g\left(x_{2}, x_{3}\right)$ it follows that every partial derivative of $S_{4}$ is zero when either $x_{2}$ or $x_{3}$ is zero. Hence for every pair of positive integers $p, q$ a function $S_{p q}$ can be defined which has the same properties of continuity and analyticity that $S_{4}$ itself has and satisfies the equation

$$
S_{4}=x_{2}^{p} x_{3}^{q} S_{p q} \text {. }
$$

In other words, $S_{4}$ admits of the factor $x_{2}{ }^{p} x_{3}{ }^{q}$ for arbitrary $p$ and $q$. This fact can readily be established by taking the factors $x_{2}$ and $x_{3}$ out of $S_{4}$ one at a time, e.g., for $x_{2} \neq 0$ the function $S_{4} / x_{2}$ has all the properties of continuity and of analyticity that $S_{4}$ itself has. The limits as $x_{2} \rightarrow 0$ of $S_{4} / x_{2}$ and all its partial derivatives exist and are zero. Hence $S_{4} / x_{2}$ defines $S_{10}$.

Now let us consider the system of ordinary differential equations

$$
\begin{aligned}
\frac{d f}{d t} & =f\left(m_{4}+S_{3}\right)+S_{4}, \\
\frac{d x_{2}}{d t} & =x_{2}\left(m_{2}+x_{3} S_{1}\right), \\
\frac{d x_{3}}{d t} & =x_{3}\left(m_{3}+x_{2} S_{2}\right) .
\end{aligned}
$$

If we can determine a surface $f=f\left(x_{2}, x_{3}\right)$ in the $f, x_{2}, x_{3}$ space which is made up of integral curves of the system of ordinary differential equations (40), such that $f\left(x_{2}, x_{3}\right)$ is of class $C^{\infty}$ in a neighborhood of $x_{2}=x_{3}=0$, and is analytic at any interior point of this neighborhood for which $x_{2} \neq 0, x_{3} \neq 0$, the func- 
tion $f\left(x_{2}, x_{3}\right)$ so defined will be a solution of the partial differential equation (37) and will define by means of the equation $f_{1}=f+g$ a function $f_{1}\left(x_{2}, x_{3}\right)$ which will be a solution of the partial differential equation (35). Thus if we can find such an $f\left(x_{2}, x_{3}\right)$ our lemma will be proved.

Suppose for the sake of definiteness that $m_{4}$ is greater than zero. We shall limit ourselves to a closed region $R$ about $f=x_{2}=x_{3}=0$ in which $\left|x_{i}\right|<1, i=2,3, m_{4}+S_{3}$ and $m_{2}+x_{3} S_{1}$ are greater than zero, and $m_{3}+x_{2} S_{2}$ is less than zero. We shall suppose that $R$ is further limited so that for $\left(f, x_{2}, x_{3}\right)$ any point in this region the functions $S_{i}$ are analytic in $f$, of class $C^{\infty}$ in $x_{2}, x_{3}$, and are analytic in $x_{2}, x_{3}$ if $x_{2} \neq 0, x_{3} \neq 0$. That this is possible follows from the properties of the $S_{i}$ stated above. The following notation will be adopted for this region $R$ :

$$
\begin{aligned}
& M_{1}=\max \left(m_{4}+S_{3}\right), M_{2}=\max \left(m_{2}+x_{3} S_{1}\right), M_{3}=\max \left(m_{3}+x_{2} S_{2}\right), \\
& N_{1}=\min \left(m_{4}+S_{3}\right), N_{2}=\min \left(m_{2}+x_{3} S_{1}\right), N_{3}=\min \left(m_{3}+x_{2} S_{2}\right) .
\end{aligned}
$$

Now consider the surface defined by the integral curves of the differential equations (40) which pass through the line

$$
f=0, x_{2}=x_{3}=\tau, \tau \geqq 0,
$$

and suppose that $t=0$ on this line. The equations of this surface may be written as

$$
f=f_{0}(t, \tau), x_{2}=x_{2}(t, \tau), x_{3}=x_{3}(t, \tau),
$$

where the functions appearing on the right hand sides of these equations, on account of the analytic properties of the $S_{i}$, are analytic in their arguments so long as $\tau \neq 0$. Until further notice, it will be understood that the discussion from now on is relative to that quadrant of $R$ for which $x_{2}$ and $x_{3}$ are positive. We wish first of all to show that there is an open region $R_{0}$ of the $x_{2}, x_{3}$ space which is bounded by the axes $x_{2}=0, x_{3}=0$ and by the arc of a circle, having its center at the origin and its radius different from zero, and is of such a nature that, when $\left(x_{2}, x_{3}\right)$ is any point in it, the last two equations of (43) can be solved for $t$ and $\tau$ as single-valued functions of $x_{2}$ and $x_{3}$. For such a region these functions $t=t\left(x_{2}, x_{3}\right)$ and $\tau=\tau\left(x_{2}, x_{3}\right)$ will evidently be analytic, and when they are substituted in the first equation of (43) we shall have $f=f\left(x_{2}, x_{3}\right)$ where $f\left(x_{2}, x_{3}\right)$ is an analytic function of its arguments for $\left(x_{2}, x_{3}\right)$ in $R_{0}$.

To this end let us find how the $t$ of any point of the surface (43) depends on the corresponding $\tau$. From the second equation of (40) we evidently have

$$
\log x_{2}-\log \tau=\int_{0}^{t}\left(m_{2}+x_{3} S_{1}\right) d t .
$$


Since $x_{2}<1$ on account of the hypotheses concerning the region $R$, for $t \geqq 0$ we may write $-\log \tau \geqq N_{2} t$ where $N_{2}$ is defined in (41). A corresponding inequality exists for $t \leqq 0$, i.e., $-\log \tau \geqq M_{3} t$, so that we know that

$$
|t| \leqq n|\log \tau|,
$$

where $n$ is the greater of $1 / N_{2}$ and $-1 / M_{3}$.

Now for $\tau \neq 0$, and $\left(f, x_{2}, x_{3}\right)$ in $R$, the partial derivatives $\partial f / \partial \tau, \partial x_{2} / \partial \tau$, and $\partial x_{3} / \partial \tau$ satisfy the equations of variation

$$
\begin{aligned}
& \frac{\partial}{\partial t} \cdot \frac{\partial f}{\partial \tau}=\frac{\partial f}{\partial \tau}\left(m_{4}+\omega T_{11}\right)+\frac{\partial x_{2}}{\partial \tau} \omega T_{12}+\frac{\partial x_{3}}{\partial \tau} \omega T_{13}, \\
& \frac{\partial}{\partial t} \cdot \frac{\partial x_{2}}{\partial \tau}=\frac{\partial f}{\partial \tau} \omega T_{21}+\frac{\partial x_{2}}{\partial \tau}\left(m_{2}+\omega T_{22}\right)+\frac{\partial x_{3}}{\partial \tau} \omega T_{33}, \\
& \frac{\partial}{\partial t} \cdot \frac{\partial x_{3}}{\partial \tau}=\frac{\partial f}{\partial \tau} \omega T_{31}+\frac{\partial x_{2}}{\partial \tau} \omega T_{32}+\frac{\partial x_{3}}{\partial \tau}\left(m_{3}+\omega T_{33}\right),
\end{aligned}
$$

where $\omega=x_{2}^{\lambda-1} x_{3}^{\lambda-1}$ and the $T_{i j}$, since every $S_{i}$ has $x_{2}{ }^{\lambda} x_{3}{ }^{\lambda}$ as a factor, are defined by the equations

$$
\begin{array}{lll}
\omega T_{11}=S_{3}+f \partial S_{3} / \partial f, \omega T_{12}=f \partial S_{3} / \partial x_{2}+\partial S_{4} / \partial x_{2}, & \omega T_{13}=f \partial S_{3} / \partial x_{3}+\partial S_{4} / \partial x_{3}, \\
\omega T_{21}=x_{2} x_{3} \partial S_{1} / \partial f, & \omega T_{22}=x_{3} S_{1}+x_{2} x_{3} \partial S_{1} / \partial x_{2}, & \omega T_{23}=\partial\left(x_{2} x_{3} S_{1}\right) / \partial x_{3}, \\
\omega T_{31}=x_{2} x_{3} \partial S_{2} / \partial f, & \omega T_{32}=\partial\left(x_{3} x_{2} S_{2}\right) / \partial x_{2}, & \omega T_{33}=x_{2} S_{2}+x_{3} x_{2} \partial S_{2} / \partial x_{8} .
\end{array}
$$

Now for $t=0, \partial f / \partial \tau=0, \partial x_{2} / \partial \tau=\partial x_{3} / \partial \tau=1$. With this in mind let us set

$$
\begin{aligned}
& \xi=\exp \left[\int_{0}^{t}\left(m_{0}+\omega T_{22}\right) d t\right], \eta=\exp \left[\int_{0}^{t}\left(m_{3}+\omega T_{33}\right) d t\right], \\
& u=\partial f / \partial \tau, \quad v+\xi=\partial x_{2} / \partial \tau, \quad w+\eta=\partial x_{3} / \partial \tau .
\end{aligned}
$$

Hence, when $t=0, y=v=w=0$. Now make use of the transformation (46) in the equations (45) and obtain

Hence

$$
\begin{aligned}
& \frac{\partial u}{\partial t}=u\left(m_{4}+\omega T_{11}\right)+(v+\xi) \omega T_{12}+(w+\eta) \omega T_{13}, \\
& \frac{\partial v}{\partial t}=u \omega T_{21}+v\left(m_{2}+\omega T_{22}\right)+(w+\eta) \omega T_{23}, \\
& \frac{\partial w}{\partial t}=u \omega T_{81}+(v+\xi) \omega T_{32}+w\left(m_{3}+\omega T_{33}\right) .
\end{aligned}
$$




$$
\begin{aligned}
& u=\exp \left[\int_{0}^{t}\left(m_{4}+\omega T_{11}\right) d t\right] \int_{0}^{t} \exp \left[-\int_{0}^{t}\left(m_{4}+\omega T_{11}\right) d t\right] \\
& v=\xi \int_{0}^{t} \xi^{-1}\left[u \omega T_{21}+(w+\eta) \omega T_{23}\right] d t \\
& w=\eta \int_{0}^{t} \eta^{-1}\left[u \omega T_{31}+(v+\xi) \omega T_{32}\right] d t .
\end{aligned}
$$

We shall limit the discussion for the time being to that part of the first quadrant of the $x_{2} x_{3}$ plane which corresponds to $t \geqq 0$ since an argument similar to that about to be given can be made when $t \leqq 0$. Define $U=\max |u|$ for $\tau$ fixed and $t$ variable, and make corresponding definitions for $V$ and $W$. Also define the constants $M_{i j}$ so that $\infty>M_{i j}>n \max \left|x_{2}^{\lambda-1} T_{i j}\right|$ in $R$, where $n$ is that in (44). Since $x_{3}$ decreases when $t$ increases, we have for this region that $x_{3}$ is less than its corresponding $\tau$. From the first equation of (48) we obtain

$$
\begin{aligned}
U & \leqq e^{M_{1} t}\left[\left\{V M_{12}+W M_{13}\right\} \tau^{\lambda-1}+\left\{e^{M_{2} t} M_{12}+M_{13}\right\} \tau^{\lambda-1}\right]|\log \tau| \\
& \leqq e^{n M_{1}|\log \tau|}\left[V M_{12}+W M_{13}+e^{\left.n M_{2}|\log \tau| M_{12}+M_{13}\right] \tau^{\lambda-1}|\log \tau|}\right. \\
& \leqq \tau^{-n M_{1}+\lambda-1}|\log \tau|\left[V M_{12}+W M_{13}+\tau^{\left.-n M_{2} M_{12}+M_{13}\right]}\right.
\end{aligned}
$$

In a similar manner from the other two equations of (48) we obtain

$$
\begin{aligned}
& V \leqq \tau^{-n M_{2}+\lambda-1}|\log \tau|\left[U M_{21}+W M_{23}+M_{23}\right], \\
& W \leqq \tau^{n N_{3}+\lambda-1}|\log \tau|\left[U M_{31}+V M_{32}+\tau^{-n M_{2} M_{32}}\right] .
\end{aligned}
$$

For $t \geqq 0$, if $\lambda$ were to satisfy the inequality

$$
\lambda>3+n\left(M_{1}+M_{2}-N_{3}\right)
$$

we could show that $U, V$ and $W$ approach zero as $\tau \rightarrow 0$ and hence that the partial derivatives $\partial x_{2} / \partial \tau$ and $\partial x_{3} / \partial \tau$ are greater than zero for $\tau$ less than a certain number which is greater than zero.

The inequality which corresponds to (51) for $t \leqq 0$ is the same as (51). Thus it follows from the definition of $n$ and from (51) that it would be sufficient if $\lambda$ were to satisfy the inequality

$$
\lambda>3+\left(M_{1}+M_{2}-N_{3}\right)\left(1 / N_{2}-1 / M_{3}\right) .
$$

But $M_{1}, M_{2}$ and $N_{3}$ depend to some extent on the choice of $\lambda$. Let us choose $\lambda$ so large that

$$
\lambda>3+L\left(m_{4}+m_{2}-m_{3}\right)\left(1 / n_{2}-1 / m_{3}\right) *
$$

* $\lambda$ will also have to satisfy another inequality which arises from the determination of the other invariant surface. 
where $L$ is any positive number greater than 1 , and then choose $R$ so that (52) holds, which evidently can be done.

But, since $\tau<1$, the $\max |\log \tau| \tau$ for the region $R$ is when $\tau=e^{-1}$. Thus for $\tau<e^{-1}$ we may write the inequalities (49) and (50) as

$$
\begin{aligned}
U & \leqq \tau\left(V M_{12}+W M_{13}\right)+\tau\left(M_{12}+M_{13}\right), \\
V & \leqq \tau\left(U M_{21}+W M_{23}\right)+\tau M_{23}, \\
W & \leqq \tau\left(U M_{81}+V M_{32}\right)+\tau M_{32} .
\end{aligned}
$$

These inequalities may be written in the form

$$
\begin{aligned}
& U-\tau M_{12} V-\tau M_{13} W \leqq \tau\left(M_{12}+M_{18}\right), \\
&-\tau M_{21} U+V-\tau M_{23} W \leqq \tau M_{23}, \\
&-\tau M_{31} U-\tau M_{32} V+W \leqq \tau M_{32} .
\end{aligned}
$$

Since the determinant of the coefficients of $U, V, W$ in the left hand members of these inequalities is definitely positive for $\tau$ small enough and since each of the right hand members contains $\tau$ as a factor and the $M_{i j}$ are constants independent of $\tau$, we see that for $\tau$ small enough $U, V$ and $W$ are as small as we desire.

This argument, with exception of that part of it relative to the choice of $\lambda$, has been made for $t \geqq 0$. A similar argument can be made for $t \leqq 0$ and hence as is seen from the equations (46), for $\tau$ small enough $\partial x_{2} / \partial \tau$ and $\partial x_{3} / \partial \tau$ are greater than zero for $\left(f, x_{2}, x_{3}\right)$ in $R$ and, as is still assumed, $x_{2}>0$ and $x_{3}>0$.

Now examine the jacobian for the last two equations of (43). In $R$ and for $x_{2}>0$ and $x_{3}>0$ we have always $\partial x_{2} / \partial t$ greater than zero and $\partial x_{3} / \partial t$ less than zero. From these facts and those proved in the preceding paragraphs we see that we can find an open region $R_{0}$ defined by $x_{2}>0, x_{3}>0, x_{2}{ }^{2}+x_{3}{ }^{2}$ $<2 \tau_{0}^{2}$ where $\tau_{0}$ is a constant greater than zero but so small that the jacobian

$$
\left|\begin{array}{ll}
\partial x_{2} / \partial \tau & \partial x_{3} / \partial \tau \\
\partial x_{2} / \partial t & \partial x_{3} / \partial t
\end{array}\right|
$$

is always less than zero. Hence for this region $R_{0}$ we can always solve the last two equations of (43) for $t$ and $\tau$ as analytic functions of $x_{2}$ and $x_{3}$ and thus obtain $f=f\left(x_{2}, x_{3}\right)$ as has already been described.

We wish now to show that the limits of $f\left(x_{2}, x_{3}\right)$ and all its derivatives as $x_{2} \rightarrow 0$ or as $x_{3} \rightarrow 0$ are zero. To do this let us note that proving any function $G\left(x_{2}, x_{3}\right)$ satisfies the relationship

$$
G\left(x_{2}, x_{3}\right)=O\left(x_{2}^{p} x_{3}^{q}\right)
$$


for an arbitrary pair of positive integers $p, q$ is equivalent to proving that it satisfies the relationship

$$
G\left(x_{2}, x_{3}\right)=O\left(\tau^{r}\right)
$$

for an arbitrary positive integer $r$. It is understood that $x_{2}$ and $x_{3}$ are positive and that $\tau$ is the value of the parameter which corresponds to the point $\left(x_{2}, x_{3}\right)$ by means of the last two equations of (43).

First of all let us observe that the last two equations of (40) imply that

$$
\tau / x_{2} \leqq \tau^{-n M_{2}}, \quad \tau / x_{3} \leqq \tau^{n N_{2}} .
$$

Now consider that part of the quadrant in the $x_{2} x_{3}$ plane which corresponds to $t \geqq 0$. For this region $x_{3} \leqq \tau$, and the equation (54) implies the existence of a positive constant $M_{q}$ such that $\left|G\left(x_{2}, x_{3}\right)\right| \leqq x_{3}{ }^{q} M_{q}$, where $q$ is any positive integer. Hence $\left|G\left(x_{2}, x_{3}\right)\right| \leqq \tau^{q} M_{q}$. In the same way we can show that for that part of the quadrant which corresponds to $t \leqq 0$ there exists a positive constant $M_{p}$ corresponding to any arbitrary positive integer $p$ such that $\left|G\left(x_{2}, x_{3}\right)\right| \leqq \tau^{p} M_{p}$. If we define $M_{p q}$ as the greater of $M_{p}$ and $M_{q}$ we see that for $t$ positive or negative

$$
\left|G\left(x_{2}, x_{3}\right)\right| \leqq \tau^{r} M_{r r}
$$

for an arbitrary positive integer $r$, the $M_{r r}$ being a positive constant depending on the $r$. Hence the relationship (54) implies the relationship (55).

Now consider the converse situation. The equation (55) implies the existence of a positive constant $M_{r r}$ and a relationship of the form (57) for an arbitrary positive integer $r$. On using the inequalities (56) we obtain the inequalities

$$
\frac{|G|}{x_{2} x_{3}^{q}} \leqq \frac{\tau^{p}}{x_{2}^{p}} \cdot \frac{\tau^{q}}{x_{3}^{q}} M_{r r} \tau^{r-p-q} \leqq M_{r r} \tau^{r-p-q-p n M_{2}+q n N_{3}} .
$$

If we choose $r$ so large that $r-p-q-p n M_{2}+q n N_{3}>0$, the quantity after the last inequality sign is finite and hence we have the result that the relationship (55) implies the relationship (54).

We are now in a position to discuss the behavior of $f\left(x_{2}, x_{3}\right)$ and of its partial derivatives as $x_{2} \rightarrow 0$ or as $x_{3} \rightarrow 0$. From the first equation of (40) and from (39) and (42) we have that

whence

$$
f=\exp \left[\int_{0}^{t}\left(m_{4}+S_{3}\right) d t\right] \int_{0}^{t} \exp \left[-\int_{0}^{t}\left(m_{4}+S_{3}\right) d t\right] O\left(\tau^{r}\right) d t,
$$

$$
|f| \leqq \tau^{-n M_{1}}\left|O\left(\tau^{r}\right)\right| n|\log \tau| \leqq\left|O\left(\tau^{r-n M_{2}-1}\right)\right|
$$

for $r$ an arbitrary positive integer. Hence $f\left(x_{2}, x_{3}\right)=O\left(x_{2}{ }^{p} x_{3}{ }^{q}\right)$ for arbitrary positive integers $p, q$. 
In order to discuss the behavior of $\partial f / \partial x_{2}$ as $x_{2} \rightarrow 0$ or as $x_{3} \rightarrow 0$ we shall make use of the fact that

$$
\frac{\partial f}{\partial x_{2}}=\frac{\partial f}{\partial t} \cdot \frac{\partial t}{\partial x_{2}}+\frac{\partial f}{\partial \tau} \cdot \frac{\partial \tau}{\partial x_{2}} .
$$

Since $f\left(x_{2}, x_{3}\right)=O\left(x_{2}{ }^{p} x_{3}{ }^{a}\right)$ for arbitrary $p, q$ as has just been proved, the first equation of (40) shows that $\partial f / \partial t$ has the same property. Now consider the equations (45) once again. From them. we have proved that $\partial x_{2} / \partial \tau$ behaves like $\xi$ of (46) for $\tau$ small enough and hence for $x_{2}$ or $x_{3}$ small enough. Hence $\partial x_{2} / \partial \tau$, as $x_{2} \rightarrow 0$ or as $x_{3} \rightarrow 0$, is at most infinite like $\tau^{-n M_{2}}$. Similarly we have $\partial x_{3} / \partial \tau=O\left(\tau^{n N_{3}}\right)$. Now we are in a position to examine the behavior of $\partial \tau / \partial x_{2}$ and of $\partial t / \partial x_{2}$. To do this let us consider the identities

and

$$
\begin{aligned}
& 1=\frac{\partial \tau}{\partial x_{2}} \cdot \frac{\partial x_{2}}{\partial \tau}+\frac{\partial \tau}{\partial x_{3}} \cdot \frac{\partial x_{3}}{\partial \tau}, \\
& 0=\frac{\partial \tau}{\partial x_{2}} \cdot \frac{\partial x_{2}}{\partial t}+\frac{\partial \tau}{\partial x_{3}} \cdot \frac{\partial x_{3}}{\partial t},
\end{aligned}
$$

Since the determinant

$$
\begin{aligned}
& 1=\frac{\partial t}{\partial x_{2}} \cdot \frac{\partial x_{2}}{\partial t}+\frac{\partial t}{\partial x_{8}} \cdot \frac{\partial x_{3}}{\partial t}, \\
& 0=\frac{\partial t}{\partial x_{2}} \cdot \frac{\partial x_{2}}{\partial \tau}+\frac{\partial t}{\partial x_{3}} \cdot \frac{\partial x_{3}}{\partial \tau} .
\end{aligned}
$$

$$
\left|\begin{array}{ll}
\partial x_{2} / \partial \tau & \partial x_{3} / \partial \tau \\
\partial x_{2} / \partial t & \partial x_{3} / \partial t
\end{array}\right|
$$

is zero only when $x_{2}=x_{3}=0$ and then vanishes like $\tau$, we see that $\partial t / \partial x_{2}$ and $\partial \tau / \partial x_{2}$ are at most infinite like a finite power of $\tau^{-1}$ as $x_{2} \rightarrow 0$ or as $x_{3} \rightarrow 0$. Let this power be $m$ so that we have that $\partial t / \partial x_{2}$ and $\partial \tau / \partial x_{2}$ are $O\left(\tau^{-m}\right)$ where $m$ is some finite positive number.

Now return to the first differential equation of (45) and note that in addition to the facts used in the discussion there made $T_{12}$ and $T_{13}$ are $O\left(x_{2}{ }^{p} x_{3}{ }^{q}\right)$ for arbitrary positive integers $p, q$. On integrating this equation and using the inequalities just established we obtain the result that

$$
\begin{aligned}
\left|\frac{\partial f}{\partial \tau}\right|=\mid & \exp \left[\int_{0}^{t}\left(m_{4}+\omega T_{11}\right) d t\right] \int_{0}^{t} \exp \left[-\int_{0}^{t}\left(m_{4}+\omega T_{11}\right) d t\right] \\
& \cdot\left[\frac{\partial x_{2}}{\partial \tau} \omega T_{12}+\frac{\partial x_{3}}{\partial \tau} \omega T_{13}\right] d t\left|\leqq \tau^{-n M_{1}}\right| O\left(\tau^{p}\right)|n| \log \tau|=| O\left(\tau^{r}\right) \mid
\end{aligned}
$$

for an arbitrary positive integer $r$. 
Out of this discussion and the equation (58) follows immediately the fact that $\partial f / \partial x_{2}=O\left(\tau^{r}\right)=O\left(x_{2}{ }^{p} x_{3}{ }^{a}\right)$ where $r, p$ and $q$ are arbitrary positive integers. It is evident by induction, using the equations (40), (59) and (60), that corresponding statements are true for all the partial derivatives of $f\left(x_{2}, x_{3}\right)$ with respect to $x_{2}$ and $x_{3}$ and hence that all these partial derivatives are zero for $x_{2}=0$ or for $x_{3}=0$.

Although the function $f\left(x_{2}, x_{3}\right)$ which we have just been discussing has been defined only for the quadrant of $R$ for which $x_{2}$ and $x_{3}$ are positive, it is readily seen that it can be defined in the other quadrants in a similar manner and will have the same properties with respect to these quadrants as it has with respect to the first one. When these four functions are joined along the axes they unite to form a function which is of class $C^{\infty}$ in a neighborhood of the origin, is analytic for $x_{2} \neq 0, x_{3} \neq 0$, and is zero together with all its partial derivatives when $x_{2}=0$ or when $x_{3}=0$. This completes the proof of Lemma 3.

\section{FirST NON-ANALYTIC NORMaLIZATION}

Thus we have found two invariant surfaces other than $x_{3}=0$. For purposes of notation let the equations of these invariant surfaces be

$$
\begin{aligned}
& x_{1}=x_{2}^{2} x_{3}^{2} f_{1}\left(x_{2}, x_{3}\right)=F\left(x_{2}, x_{3}\right), \\
& x_{2}=x_{1}^{2} x_{3}^{2} g_{1}\left(x_{1}, x_{3}\right)=G\left(x_{1}, x_{3}\right) .
\end{aligned}
$$

Reviewing the properties of $F$ and $G$, we know that the function $F\left(x_{2}, x_{3}\right)$ is of class $C^{\infty}$ in a neighborhood $\left|x_{2}\right|,\left|x_{3}\right|<r_{f}, r_{f}>0$ of $x_{2}=x_{3}=0$ and is analytic at any point $\left(x_{2}, r_{3}\right)$ of this neighborhood for which $x_{2} \neq 0, x_{3} \neq 0$; the function $G\left(x_{1}, x_{3}\right)$ is of class $C^{\infty}$ in a certain neighborhood, $\left|x_{1}\right|,\left|x_{3}\right|<r_{0}, r_{0}>0$, of $x_{1}=x_{3}=0$ and is analytic at a point $\left(x_{1}, x_{3}\right)$ of this neighborhood for which $x_{1} \neq 0, x_{3} \neq 0$. Let $r_{0}$ be the smaller of $r_{f}$ and $r_{g}$.

The aim of this section is to use these invariant surfaces in a transformation which will transform the differential equations (30) into ones of the same form in which the $Q_{1}=Q_{2}=0$. Now the invariant surfaces (61) fail to be analytic along the axes and we wish to set up the equations of a transformation which will carry these surfaces into coördinate planes, and will be analytic except possibly for those points which lie on these surfaces. To this end let us examine the transformation

$$
\begin{aligned}
& x_{1}=y_{1}+F\left[x_{2}-G\left(y_{1}, y_{3}\right), y_{3}\right], \\
& x_{2}=y_{2}+G\left[x_{1}-F\left(y_{2}, y_{3}\right), y_{3}\right], \\
& x_{3}=y_{3},
\end{aligned}
$$

and confine our attention in the $x_{i}$ space to a region $R_{x}$ about the origin for 
which $\left|x_{j}\right|<r_{0}$, and in the $y_{i}$ space to a region $R_{y}$ about the origin for which $\left|y_{i}\right|<r_{0}$. Furthermore, let $R_{x}$ and $R_{y}$ be so chosen that the transformation (62) establishes a one-to-one correspondence between the points of $R_{x}$ and those of $R_{y}$, which clearly can be done so that the origin $\left(x_{1}, x_{2}, x_{3}\right)=(0,0,0)$ is interior to $R_{x}$ and the origin $\left(y_{1}, y_{2}, y_{3}\right)=(0,0,0)$, which happens to correspond to the origin in the $x_{i}$ space, is interior to $R_{y}$. From the properties of $F\left(x_{2}, x_{3}\right)$ and of $G\left(x_{1}, x_{3}\right)$ it follows that when $y_{1}=0, x_{1}=F\left(x_{2}, x_{3}\right)$, and when $y_{2}=0, x_{2}=G\left(x_{1}, x_{3}\right)$. Moreover, this transformation (62) is of class $C^{\infty}$ and can fail to be analytic only when $y_{1}=0, y_{2}=0$, or $y_{3}=0$.

On solving the equations (62) for $x_{i}$ we obtain

$$
x_{1}=f_{1}\left(y_{1}, y_{2}, y_{3}\right), x_{2}=f_{2}\left(y_{1}, y_{2}, y_{3}\right), x_{3}=y_{3},
$$

where the functions $f_{i}$ are of class $C^{\infty}$ in $R_{y}$ and are analytic at any point of $R_{y}$ for which $y_{1} \neq 0, y_{2} \neq 0, y_{3} \neq 0$. Furthermore, in any one of the surfaces $y_{1}=0, y_{2}=0$, or $y_{3}=0$, the functions $f_{i}$ are of class $C^{\infty}$ in the variables of the surface, analytic at any point which does not lie on either of the axes in the surface, and are analytic along either axis in the variable of the axis in a neighborhood of the origin. This statement is obvious for $y_{3}=0$. For $y_{1}=0$, $x_{1}=F\left(y_{2}, y_{3}\right)$ and $x_{2}=y_{2}$. On account of the properties of the $F\left(x_{2}, x_{3}\right)$ the statement is seen to be true when $y_{1}=0$. In a similar manner it can be shown to be true when $y_{2}=0$.

Since the $f_{i}$ are of class $C^{\infty}$ in the $R_{y}$ we may formally expand these functions as power series in $y_{3}$ or as power series in $y_{1}$ and $y_{2}$ together. Let these expansions be written as

$$
\begin{aligned}
x_{i} & \sim \alpha_{i 0}\left(y_{1}, y_{2}\right)+\alpha_{i 1}\left(y_{1}, y_{2}\right) y_{3}+\cdots \\
& =\alpha_{i 00}\left(y_{3}\right)+\alpha_{i 10}\left(y_{3}\right) y_{1}+\alpha_{i 01}\left(y_{3}\right) y_{2}+\cdots,
\end{aligned}
$$

$i=1,2$. We shall now prove

LEMMA 4. The $\alpha_{i j}\left(y_{1}, y_{2}\right)$ of (64) are analytic in $y_{1}, y_{2}$ for $\left|y_{1}\right|,\left|y_{2}\right|<r_{0}$, and the $\alpha_{i j k}\left(y_{3}\right)$ are analytic in $y_{3}$ for $\left|y_{3}\right|<r_{0}$.

Since $F\left(x_{2}, x_{3}\right)$ and $G\left(x_{1}, x_{3}\right)$ each contains $x_{3}{ }^{2}$ as a factor, it is seen that $\alpha_{i 0}=y_{i}, i=1,2$. We shall denote the partial derivative of $F$ with respect to its first argument by $F_{1}$, and with respect to its second by $F_{2}$. Furthermore, when the arguments of $F$ are $y_{2}, y_{3}$ we shall write it merely as $F$, but when the arguments are $x_{2}-G\left(y_{1}, y_{3}\right), y_{3}$ we shall write it as $F^{*}$. Corresponding notations will be adopted for $G$ and its partial derivatives.

Now differentiate the first two equations of (62) partially with respect to $y_{3}$. Hence 


$$
\frac{\partial x_{1}}{\partial y_{3}}=F_{2}^{*}+F_{1}^{*}\left(\frac{\partial x_{2}}{\partial y_{3}}-G_{2}\right), \quad \frac{\partial x_{2}}{\partial y_{3}}=G_{2}^{*}+G_{1}^{*}\left(\frac{\partial x_{1}}{\partial y_{3}}-F_{2}\right),
$$

whence

$$
\begin{aligned}
& \frac{\partial x_{1}}{\partial y_{3}}\left(1-F_{1}^{*} G_{1}^{*}\right)=F_{2}^{*}+F_{1}^{*} G_{2}^{*}-F_{1}^{*} G_{1}^{*} F_{2}-F_{1}^{*} G_{2}, \\
& \frac{\partial x_{2}}{\partial y_{3}}\left(1-F_{1}^{*} G_{1}^{*}\right)=G_{2}^{*}+G_{1}^{*} F_{2}^{*}-G_{1}^{*} F_{1}^{*} G_{2}-G_{1}^{*} F_{2} .
\end{aligned}
$$

Now for $y_{3}=0, F_{1} G_{1}$ and the right hand sides of the equations (65) are zero since $F$ and $G$ both contain $x_{3}{ }^{2}$ as a factor. Hence $\partial x_{1} / \partial y_{3}$ and $\partial x_{2} / \partial y_{3}$ are both zero for $y_{3}=0$.

Now recall the fact that $F$, as well as all its partial derivatives, is analytic in $x_{2}$ for $\left|x_{2}\right|<r_{0}, x_{3}=0$, and that $G$ and its partial derivatives have corresponding properties. Hence any partial derivative of any order of $F^{*}$ or $G^{*}$ with respect to the arguments $x_{2}-G, y_{3}$, and $x_{1}-F, y_{3}$, respectively, is analytic in $y_{2}$ and $y_{1}$, respectively, for $\left|y_{i}\right|<r_{0}, y_{3}=0$, since $y_{1}=x_{1}$ and $y_{2}=x_{2}$ for $y_{3}=0$. The $n$th order partial derivatives of $F_{i}{ }^{*}$ and $G_{i}{ }^{*}$ with respect to $y_{3}$ are polynomials in the partial derivatives of $x_{1}$ and $x_{2}$ with respect to $y_{3}$ up to and including those of the $n$th order. The coefficients in these polynomials are polynomials in $F_{j}{ }^{*}, G_{j}{ }^{*}$, and their partial derivatives with respect to their respective arguments. Hence, from the equations (65) we may determine seriatim all the partial derivatives of $x_{1}$ and $x_{2}$ with respect to $y_{3}$ at $y_{3}=0$ as functions of $y_{1}, y_{2}$ which are analytic for $\left|y_{1}\right|,\left|y_{2}\right|<r_{0}$.

Now differentiate the first two equations of (62) partially with respect to $y_{1}$ and $y_{2}$. We obtain on simplification

$$
\begin{array}{ll}
\frac{\partial x_{1}}{\partial y_{1}}\left[1-F_{1}^{*} G_{1}^{*}\right]=1-F_{1}^{*} G_{1}, & \frac{\partial x_{2}}{\partial y_{1}}=G_{1}^{*} \frac{\partial x_{1}}{\partial y_{1}}, \\
\frac{\partial x_{2}}{\partial y_{2}}\left[1-F_{1}^{*} G_{1}^{*}\right]=1-G_{1}^{*} F_{1}, & \frac{\partial x_{1}}{\partial y_{2}}=F_{1}^{*} \frac{\partial x_{2}}{\partial y_{2}} .
\end{array}
$$

Now when $y_{1}=y_{2}=0, x_{1}=x_{2}=0$. Hence, by using an argument similar to that used in connection with the partial derivatives with respect to $y_{3}$, it is seen that from (66) we may determine seriatim all the partial derivatives of $x_{1}$ and of $x_{2}$ with respect to $y_{1}$ and $y_{2}$ at $y_{1}=y_{2}=0$ as functions of $y_{3}$ which are analytic for $\left|y_{3}\right|<r_{0}$. This completes the proof of the lemma.

From the equations (65) and (66) we can now prove that all the partial derivatives of $x_{1}$ and $x_{2}$, when $y_{1}, y_{2}$, or $y_{3}$ is zero, are of class $C^{\infty}$ in the remaining variables and are analytic in these variables provided neither is zero. We shall prove this only for the $\partial x_{1} / \partial y_{3}$ for $y_{1}=0$ because an induction 
proof can be made for all the other derivatives except those of the first order and a proof can be given for them along the lines of that to follow. As we have already seen, if $y_{1}=0$, then $x_{2}=y_{2}, x_{3}=y_{3}$, and $x_{1}=F\left(y_{2}, y_{3}\right)$. Hence the coefficient of $\partial x_{1} / \partial y_{3}$ in the first equation of (65) is 1 for $y_{1}=0$, and it is readily verified that the right hand member of this equation is of class $C^{\infty}$ in $y_{2}$ and $y_{3}$, and is analytic in these variables for $y_{2} \neq 0, y_{3} \neq 0$.

Now let us perform the transformation of variables defined by the equations (62) or the equations (63). The differential equations (30) will be transformed into differential equations of the same form in $y_{i}$ since under the transformation (62) the $x_{1}=x_{2}=0$ axis and the $x_{3}=0$ plane are invariant. From the definition of $y_{1}=0$ it follows that when an integral curve has at any point $y_{1}=0$, it has $y_{1}=0$ for all its points in the neighborhood of the origin under consideration. A similar statement is true concerning $y_{2}$. Hence the right hand members of the first two equations in our new system have $y_{1}$ and $y_{2}$, respectively, as factors. Thus the new equations have the form

$$
\begin{aligned}
& \frac{d y_{1}}{d t}=y_{1}\left(m_{1}+y_{3} U_{1}\right), \\
& \frac{d y_{2}}{d t}=y_{2}\left(m_{2}+y_{3} U_{2}\right), \\
& \frac{d y_{3}}{d t}=y_{3}\left(m_{3}+y_{1} U_{3}+y_{2} U_{4}\right),
\end{aligned}
$$

in which the $U_{i}$ are functions of the $y_{i}$ which are of class $C^{\infty}$ in a neighborhood of the origin and are analytic at any point of this neighborhood for which $y_{i} \neq 0, j=1,2,3$. Furthermore, the $U_{i}$ and all their partial derivatives have the property that in any one of the coördinate planes $y_{j}=0, j=1,2,3$, they are of class $C^{\infty}$ in a neighborhood of the origin, analytic at any point of this neighborhood which does not lie on the coördinate axes of this plane, and analytic along any one of these coördinate axes in the variable of the axis in a neighborhood of the origin. All these statements follow at once from the properties of the transformation (63), because on differentiating the equations of (63) we obtain

$$
\begin{aligned}
& \frac{d x_{1}}{d t}=\frac{\partial f_{1}}{\partial y_{1}} \cdot \frac{d y_{1}}{d t}+\frac{\partial f_{1}}{\partial y_{2}} \cdot \frac{d y_{2}}{d t}+\frac{\partial f_{1}}{\partial y_{3}} \cdot \frac{d y_{3}}{d t}=x_{1}\left(m_{1}+x_{3} P_{1}\right)+x_{2}^{2} x_{3}^{2} Q_{1} \\
& \frac{d x_{2}}{d t}=\frac{\partial f_{2}}{\partial y_{1}} \cdot \frac{d y_{1}}{d t}+\frac{\partial f_{2}}{\partial y_{2}} \cdot \frac{d y_{2}}{d t}+\frac{\partial f_{2}}{\partial y_{3}} \cdot \frac{d y_{3}}{d t}=x_{2}\left(m_{2}+x_{3} P_{2}\right)+x_{1}^{2} x_{3}^{2} Q_{2} \\
& \frac{d x_{3}}{d t}=\frac{\partial f_{3}}{\partial y_{1}} \cdot \frac{d y_{1}}{d t}+\frac{\partial f_{3}}{\partial y_{2}} \cdot \frac{d y_{2}}{d t}+\frac{\partial f_{3}}{\partial y_{3}} \cdot \frac{d y_{3}}{d t}=x_{3}\left(m_{3}+x_{1} P_{3}+x_{2} P_{4}\right)
\end{aligned}
$$


Now replace the $x_{i}$ in these equations by the functions of $y_{j}$ as given in (63). Since all the functions of $y_{j}$ which now appear in these equations (68) as well as all their partial derivatives have the same properties of continuity and of analyticity that the $f_{i}$ themselves have, we see at once that our statement concerning the $U_{i}$ and their partial derivatives is true.

\section{SECOND NON-ANALYTIC NORMALIZATION}

The aim of this section is to reduce the system of differential equations (67) to an equivalent system of the same form for which the formal series for the $\phi_{i}$ will consist in the linear terms only. To this end we shall first prove

LEMMA 5. Consider the formal series

$$
\begin{aligned}
\psi & \sim x_{1}+\cdots+\frac{a_{m n p}}{m ! n ! p !} x_{1}{ }^{m} x_{2}{ }^{n} x_{3}{ }^{p}+\cdots \\
& =x_{1}+\alpha_{1}\left(x_{1}, x_{2}\right) x_{3}+\cdots+\alpha_{p}\left(x_{1}, x_{2}\right) x_{3}{ }^{p}+\cdots \\
& =x_{1}+\cdots+\alpha_{m n}\left(x_{3}\right) x_{1}{ }^{m} x_{2}^{n}+\cdots
\end{aligned}
$$

in which the only linear term is $x_{1}$, every other term contains $x_{1} x_{3}$ or $x_{2} x_{3}$ as a factor, $\alpha_{p}$ are analytic in $x_{1}, x_{2}$ for $\left|x_{1}\right|,\left|x_{2}\right|<r, r>0$, and $\alpha_{m n}$ are analytic in $x_{3}$ for $\left|x_{3}\right|<r$. Into the series (69) substitute the formal series

$$
\begin{aligned}
x_{i} & \sim y_{i}+\beta_{i 1}\left(y_{1}, y_{2}\right) x_{3}+\cdots+\beta_{i p}\left(y_{1}, y_{2}\right) x_{3}{ }^{p}+\cdots \\
& =y_{i}+\cdots+\beta_{i m n}\left(x_{3}\right) y_{1}^{m} y_{2}^{n}+\cdots, i=1,2,
\end{aligned}
$$

in which the only linear term in the ith series is $y_{i}$, every other term contains $y_{1} x_{3}$ or $y_{2} x_{3}$ as a factor, the $\beta_{i p}$ are analytic in $y_{1}, y_{2}$ for $\left|y_{1}\right|,\left|y_{2}\right|<r$ and $\beta_{i m n}$ are analytic in $x_{3}$ for $\left|x_{3}\right|<r$, and arrange the resulting series as

$$
\begin{aligned}
\psi & \sim \gamma_{0}\left(y_{1}, y_{2}\right)+\cdots+\gamma_{p}\left(y_{1}, y_{2}\right) x_{3}{ }^{p}+\cdots \\
& =\gamma_{00}\left(x_{3}\right)+\cdots+\gamma_{m n}\left(x_{3}\right) y_{1}{ }^{m} y_{2}{ }^{n}+\cdots .
\end{aligned}
$$

Then $\gamma_{i}$ are analytic in $y_{1}, y_{2}$ for $\left|y_{1}\right|,\left|y_{2}\right|<r$ and $\gamma_{m n}$ are analytic in $x_{3}$ for $\left|x_{3}\right|<r$.

The truth of this lemma is almost obvious. From the properties of the series (69) and (70) it follows at once that $\gamma_{0}=y_{1}$ and that $\gamma_{00}=0$. It readily follows that $\gamma_{1}=\beta_{11}\left(y_{1}, y_{2}\right)+\alpha_{1}\left(y_{1}, y_{2}\right)$ which is obviously analytic for $\left|y_{1}\right|$, $\left|y_{2}\right|<r$. Seriatim, we may prove that all the $\gamma_{p}$ are analytic for $\left|y_{1}\right|,\left|y_{2}\right|<r$, and in a similar manner we may prove that all the $\gamma_{m n}$ are analytic for $\left|x_{3}\right|<r$.

Now consider the effect of the transformation (62) on the formal series 
$\phi_{i}$. From Lemmas 4 and 5 it follows that the series $\phi_{i}$ when expanded in powers of $y_{j}$ have the property that when they are arranged as power series in $y_{3}$ the coefficients are analytic in $y_{1}$ and $y_{2}$ for $\left|y_{1}\right|,\left|y_{2}\right|<r_{0}$, and when arranged as power series in $y_{1}$ and $y_{2}$ the coefficients are analytic in $y_{3}$ for $\left|y_{3}\right|<r_{0}$. But these series in $y_{j}$ obtained by substituting the series of (64) for the $x_{j}$ in the series for the $\phi_{i}$ are the same as the formal series solutions of the partial differential equations

$$
\begin{aligned}
\frac{\partial \phi_{i}}{\partial y_{1}} y_{1}\left(m_{1}+y_{3} U_{1}\right) & +\frac{\partial \phi_{i}}{\partial y_{2}} y_{2}\left(m_{2}+y_{3} U_{2}\right) \\
& +\frac{\partial \phi_{i}}{\partial y_{3}} y_{3}\left(m_{3}+y_{1} U_{3}+y_{2} U_{4}\right)=m_{i} \phi_{i}
\end{aligned}
$$

since the equations (72) are the transforms of the equations (31) under the transformation (62). From the form of the equations (72) it follows that the formal series for $\phi_{i}$ has $y_{i}$ as a factor for $i=1,2,3$, respectively. Hence we may write

$$
\phi_{i} \sim y_{i}\left(1+\cdots+\frac{a_{i m n p}}{m ! n ! p !} y_{1}{ }^{m} y_{2}^{n} y_{3}{ }^{p}+\cdots\right) \quad(i=1,2,3),
$$

in which the series within the brackets have the same properties relative to the convergence of sets of terms as the series for the $\phi_{i}$ themselves have. Furthermore, the series for $\phi_{1}$ and $\phi_{2}$ reduce to $y_{1}$ and $y_{2}$, respectively, when $y_{3}=0$, and the series for $\phi_{3}$ reduces to $y_{3}$ when $y_{1}=y_{2}=0$. This follows immediately from the form of the partial differential equations (72).

Now "fit" the series within the parentheses appearing in the equations (73) by means of three functions $F_{i}\left(y_{1}, y_{2}, y_{3}\right)$ in the manner described in Lemma 2, leaving the constant terms as they are. Hence we have the transformation

$$
z_{i}=y_{i}\left(1+F_{i}\left(y_{1}, y_{2}, y_{3}\right)\right) \quad(i=1,2,3),
$$

defined in which the $F_{i}$ have the properties relative to the $\phi_{i}$ which are peculiar to their mode of definition.

We wish now to determine the type of the system of differential equations which the $z_{i}$ satisfy when the $y_{j}$ in the equations (74) are replaced by an arbitrary solution $y_{j}(t)$ of the differential equations (67). On account of the properties of the power series $\phi_{i}$ which were mentioned in a preceding paragraph, we see that the transformation (74) has the property that if $y_{1}=y_{2}=0$, then $z_{1}=z_{2}=0$ and $z_{3}=y_{3}$, and if $y_{3}=0$, then $z_{3}=0, z_{1}=y_{1}$ and $z_{2}=y_{2}$. Hence the form of the differential equations which the $z_{i}$ satisfy is the same as the form of the differential equations which the $y_{i}$ satisfy, since also $y_{i}=0$ implies that $z_{i}=0$ for $i=1,2,3$. 
On remembering that

$\frac{d z_{i}}{d t}=\frac{\partial z_{i}}{\partial y_{1}} y_{1}\left(m_{1}+y_{3} U_{1}\right)+\frac{\partial z_{i}}{\partial y_{2}} y_{2}\left(m_{2}+y_{3} U_{2}\right)+\frac{\partial z_{i}}{\partial y_{3}} y_{3}\left(m_{3}+y_{1} U_{3}+y_{2} U_{4}\right)$, and on recalling the definition of the $z_{j}$ we see that

$$
\frac{d z_{i}}{d t}-m_{i} z_{i}=R_{i} \quad(i=1,2,3),
$$

where the $R_{i}$ are functions of the $y_{j}$ which have the same properties as have the $F_{i}$ in a neighborhood of the origin and are zero together with all their partial derivatives for $y_{1}=y_{2}=0$ or for $y_{3}=0$. From these properties of the $\boldsymbol{R}_{\boldsymbol{i}}$ and those of the transformation (74) it follows that the functions $\boldsymbol{R}_{\boldsymbol{i}}$ and all their partial derivatives with respect to the $z_{j}$ are zero when $z_{1}=z_{2}=0$ or when $z_{3}=0$.

From this argument follows

LEMMA 6. Under the transformation (74) the system of differential equations (67) is equivalent to a system of the type

$$
\begin{aligned}
& \frac{d z_{1}}{d t}=z_{1}\left(m_{1}+z_{3} T_{1}\right), \\
& \frac{d z_{2}}{d t}=z_{2}\left(m_{2}+z_{3} T_{2}\right), \\
& \frac{d z_{3}}{d t}=z_{3}\left(m_{3}+z_{1} T_{3}+z_{2} T_{4}\right),
\end{aligned}
$$

where $T_{i}$ are functions of $z_{j}$, are of Class $C^{\infty}$ in a neighborhood of $z_{1}=z_{2}=z_{3}=0$, are analytic for $z_{1} \neq 0, z_{2} \neq 0, z_{3} \neq 0$, are analytic together with all their partial derivatives in $z_{i}, z_{j}$ for $z_{k}=0, i \neq j, i \neq k, j \neq k$, and are zero together with all their partial derivatives when $z_{1}=z_{2}=0$ or when $z_{3}=0$.

It may be noted here and will be found of use in a later section that the properties of the $T_{i}$ imply that each is $O\left[\left(z_{1}^{2}+z_{2}^{2}\right)^{p} z_{3}{ }^{q}\right]$ for any positive integers $p, q$.

\section{FinAL REDUCTION}

In this section we shall make the final reduction, i.e., we shall find a transformation which will reduce the equations (75) to the form (8). The finding of such a transformation will be effected when a certain set of three functions is determined. Since all three of these functions can be determined in exactly the same way, we shall limit ourselves to the determination of the first. 
In fact we shall prove the following lemma by showing only how to obtain the first of the functions $g_{i}$ :

LEMMA 7. There exists a transformation of the type

$$
z_{i}^{*}=z_{i} g_{i}\left(z_{1}, z_{2}, z_{8}\right)
$$$$
(i=1,2,3) \text {, }
$$

where the functions $g_{i}\left(z_{1}, z_{2}, z_{3}\right)$ are of class $C^{\infty}$ in a neighborhood of the origin and are analytic for $z_{1} \neq 0, z_{2} \neq 0, z_{3} \neq 0$, under which the differential equations (75) are equivalent to the differential equations

$$
\frac{d z_{i}^{*}}{d t}=m_{i} z_{i}^{*} \quad(i=1,2,3) .
$$

Furthermore, under this transformation the plane $z_{i}=0$ corresponds to the plane $z_{i}^{*}=0$ for $i=1,2,3$.

The discussion will be relative to a closed region $S$ of the $z_{i}$ space which contains the origin as an interior point and in which $z_{1}^{2}+z_{2}^{2}<1, z_{3}^{2}<1, m_{1}+z_{3} T_{1}$ and $m_{2}+z_{3} T_{2}$ are greater than zero and $m_{3}+z_{1} T_{3}+z_{2} T_{4}$ is less than zero. It will be further assumed that $S$ is composed only of points which lie on integral curves of the differential equations of the system (75) which intersect the cone

$$
z_{1}^{2}+z_{2}^{2}-z_{3}^{2}=0 .
$$

It is readily seen that this hypothesis concerning $S$ is compatible with the others, since, according to the others, the slopes $d z_{1} / d z_{3}$ and $d z_{2} / d z_{3}$ of any integral curve in $S$ are zero or infinite only for those integral curves lying in the $z_{3}=0$ plane or in the $z_{1}=z_{2}=0$ axis. This implies that the integral curves of the differential equations (75) passing through an arbitrary point in a sufficiently small neighborhood of the origin will intersect the cone (76) in $S$. On account of the signs of the left hand members of the equations (75) in $S$, it is seen that every integral curve in $S$ with the exception of those lying in the $z_{3}=0$ plane or in the $z_{1}=z_{2}=0$ axis intersects the cone in only one point. It will be found convenient to define $S_{0}$ as $S$ with the plane $z_{3}=0$ and the axis $z_{1}=z_{2}=0$ removed.

If we write the equations of the cone (76) in the parametric form

$$
z_{1}=\tau_{1}, \quad z_{2}=\tau_{2}, \quad z_{3}= \pm\left(\tau_{1}^{2}+\tau_{2}^{2}\right)^{1 / 2},
$$

and choose these equations as the equations giving the initial values of the $z_{i}$ when $t=0$, we see that the points in $S_{0}$ are placed in a one-to-one correspondence with the points in the corresponding $\tau_{1}, \tau_{2}, t$ space by means of the equations of the integral curves of the differential equations (75),

$$
z_{i}=z_{i}\left(\tau_{1}, \tau_{2}, t\right) \quad(i=1,2,3) .
$$


where the functions $z_{i}$ are of class $C^{\infty}$ in their arguments for $\left(z_{1}, z_{2}, z_{3}\right)$ in $S_{0}$, and are analytic if $\tau_{1} \neq 0, \tau_{2} \neq 0$.

Let us introduce the following notation relative to the region $S$ :

$M_{1}=\max \left(m_{1}+z_{3} T_{1}\right), M_{2}=\max \left(m_{2}+z_{3} T_{2}\right), M_{3}=\max \left(m_{3}+z_{1} T_{3}+z_{2} T_{4}\right)$, (79)

$N_{1}=\min \left(m_{1}+z_{3} T_{1}\right), N_{2}=\min \left(m_{2}+z_{3} T_{2}\right), N_{3}=\min \left(m_{3}+z_{1} T_{3}+z_{2} T_{4}\right)$.

Let $M_{0}$ be the larger of $M_{1}$ and $M_{2}$, and let $N_{0}$ be the smaller of $N_{1}$ and $N_{2}$.

For this region we wish to find how the $t$ of any point of $S_{0}$ varies with the $\tau_{1}^{2}+\tau_{2}^{2}$ of this point. From the first two equations of (75) it follows that

Hence

$$
\frac{d\left(z_{1}^{2}+z_{2}^{2}\right)}{z_{1}^{2}+z_{2}^{2}}=\frac{2 z_{1}^{2}\left(m_{1}+z_{3} T_{1}\right)+2 z_{2}^{2}\left(m_{2}+z_{3} T_{2}\right)}{z_{1}^{2}+z_{2}^{2}} d t .
$$

$\log \left(z_{1}^{2}+z_{2}^{2}\right)-\log \left(\tau_{1}^{2}+\tau_{2}^{2}\right)=\int_{0}^{t} \frac{2 z_{1}^{2}\left(m_{1}+z_{3} T_{1}\right)+2 z_{2}^{2}\left(m_{3}+z_{3} T_{2}\right)}{z_{1}^{2}+z_{2}^{2}} d t$.

Since $z_{1}^{2}+z_{2}^{2}$ is less than 1 in $S, \log \left(z_{1}^{2}+z_{2}^{2}\right)$ is negative. Consider that portion of $S_{0}$ which corresponds to $t \geqq 0$. For this portion $z_{1}^{2}+z_{2}^{2}$ is greater than $\tau_{1}^{2}+\tau_{2}^{2}$. Hence $t \leqq-\left[\log \left(\tau_{1}^{2}+\tau_{2}^{2}\right)\right]\left[1 /\left(2 N_{0}\right)\right]$ since $N_{0}$ is less than the integrand of the integral in the above equation.

By means of a similar argument with respect to the third equation of (75) for $t \leqq 0$ we can show that $t \geqq-\left[\log \left(\tau_{1}^{2}+\tau_{2}^{2}\right)\right]\left[1 /\left(2 N_{3}\right)\right]$. If we let $N$ be the smaller of $2 N_{0}$ and $\left|2 N_{3}\right|$ we see that

$$
|t| \leqq-\left[\log \left(\tau_{1}^{2}+\tau_{2}^{2}\right)\right][1 / N],
$$

where $\left(\tau_{1}, \tau_{2}, t\right)$ corresponds to the point $\left(z_{1}, z_{2}, z_{3}\right)$ in $S_{0}$.

Now the first equation of (75) is transformed into one of the same form by any transformation of the type

$$
z_{1}^{*}=z_{1} g_{1}\left(z_{1}, z_{2}, z_{3}\right), \quad z_{2}^{*}=z_{2}, \quad z_{3}^{*}=z_{3},
$$

where $g_{1}(0,0,0)$ is 1 . This follows from the fact that this transformation leaves the $z_{1}=0$ plane invariant. Thus we wish to determine the function $g_{1}\left(z_{1}, z_{2}, z_{3}\right)$ so that the corresponding $z_{1}^{*}$ will satisfy the equation

$$
d z_{1}^{*} / d t=m_{1} z_{1}^{*},
$$

when the $z_{i}$ in the equations (81) are replaced by an arbitrary solution $z_{i}(t)$ of (75).

On differentiating the first equation of (81) with respect to $t$ and using the fact that we wish $z_{1}^{*}$ to satisfy the equation (82), we obtain 


$$
\frac{d z_{1}^{*}}{d t}=\frac{d z_{1}}{d t} g_{1}+z_{1} \frac{d g_{1}}{d t}=z_{1} g_{1} m_{1},
$$

whence

$$
\frac{d \log g_{1}}{d t}=-z_{3} T_{1} .
$$

If we set $f_{1}=\log g_{1}$, it is evident that, if we can find a function $f_{1}=f_{1}\left(z_{1}, z_{2}, z_{3}\right)$ of class $C^{\infty}$ such that

$$
\frac{d f_{1}}{d t}=-z_{3} T_{1}
$$

along every integral curve of (75) and such that $f_{1}(0,0,0)$ is zero, we have solved our problem because in the same way in which we found $f_{1}$ we can find functions $f_{2}$ and $f_{3}$ corresponding to $z_{2}$ and $z_{3}$, respectively, in the same way that $f_{1}$ corresponds to $z_{1}$.

Now determine the solution

$$
f_{1}=h_{1}\left(\tau_{1}, \tau_{2}, t\right)
$$

of the differential equation (83) which corresponds to the solution (78) of (75) and which has the initial value $f_{1}=0$ for $t=0$. On account of the fact that the right hand members of the differential equations (75) and (83) are of class $C^{\infty}$ in their arguments and are analytic if $z_{i} \neq 0, i=1,2,3$, the function $h_{1}$ of (84) is evidently of class $C^{\infty}$ in its arguments for $\tau_{1}^{2}+\tau_{2}^{2} \neq 0$ and analytic for $\tau_{1} \neq 0, \tau_{2} \neq 0$. Since by means of the equations (78) we can express $\tau_{1}, \tau_{2}$ and $t$ as single-valued functions of $z_{i}$ of class $C^{\infty}$ for $\left(z_{1}, z_{2}, z_{3}\right)$ in $S_{0}$ which will be analytic if $z_{i} \neq 0, i=1,2,3$, we see that we may write the equation (84) as $f_{1}=f_{1}\left(z_{1}, z_{2}, z_{3}\right)$ where the function $f_{1}\left(z_{1}, z_{2}, z_{3}\right)$ will be defined only for $\left(z_{1}, z_{2}, z_{3}\right)$ in $S_{0}$, and will be of class $C^{\infty}$ in its arguments for this region, analytic if $z_{i} \neq 0, i=1,2,3$. We need now only to investigate how this function behaves as $z_{3} \rightarrow 0$ or as $\left(z_{1}, z_{2}\right) \rightarrow(0,0)$. We shall prove that it and all its partial derivatives have limits zero as $z_{3} \rightarrow 0$ or as $\left(z_{1}, z_{2}\right) \rightarrow(0,0)$ and hence can be so defined that they are of class $C^{\infty}$ for $\left(z_{1}, z_{2}, z_{3}\right)$ in a certain neighborhood of $(0,0,0)$.

Let us first observe that, on account of the similarity of the situation here to that in Section IV, it can be readily shown that proving a function $f\left(z_{1}, z_{2}, z_{3}\right)$ to be $O\left[\left(z_{1}^{2}+z_{2}^{2}\right)^{p} z_{3}{ }^{2}{ }^{2}\right]$ is equivalent to proving it $O\left[\left(\tau_{1}^{2}+\tau_{2}^{2}\right)^{r}\right]$ where $p, q$ and $r$ are arbitrary positive integers.

Now we are in a position to discuss the behavior of $f_{1}\left(z_{1}, z_{2}, z_{3}\right)$. It follows from the equation (83) and from the inequality (80) that 


$$
\left|f_{1}\right|=\left|\int_{0}^{t} O\left[\left(z_{1}^{2}+z_{2}^{2}\right)^{p_{z_{3}}{ }^{2 q}}\right] d t\right| \leqq\left|O\left[\left(\tau_{1}^{2}+\tau_{2}^{2}\right)^{r}\right]\right| \cdot\left|\log \left(\tau_{1}^{2}+\tau_{2}^{2}\right)\right| \cdot 1 / N
$$

for an arbitrary positive integer $r$, since $T_{1}=O\left[\left(z_{1}^{2}+z_{2}^{2}\right)^{p} z_{3}{ }^{2 q}\right]$ for arbitrary positive integers $p, q$. Hence $f_{1}\left(z_{1}, z_{2}, z_{3}\right)=O\left[\left(z_{1}^{2}+z_{2}^{2}\right)^{p} z_{3}^{2 q}\right]$ for arbitrary positive integers $p, q$.

By the method used in Section IV for the function $f\left(x_{2}, x_{3}\right)$ it can be shown that every partial derivative of $f_{1}\left(z_{1}, z_{2}, z_{3}\right)$ is also $O\left[\left(z_{1}^{2}+z_{2}^{2}\right)^{p} z_{3}{ }^{2} q\right]$ for arbitrary positive integers $p, q$, since all the partial derivatives $\partial z_{1} / \partial \tau_{1}$, $\partial \tau_{1} / \partial z_{1}, \partial z_{1} / \partial t$, etc., are at most infinite like a finite power of $\left(\tau_{1}^{2}+\tau_{2}^{2}\right)^{-1}$. Hence the function $f_{1}\left(z_{1}, z_{2}, z_{3}\right)$ is of class $C^{\infty}$ in a neighborhood of the origin and therefore, on account of its definition, the function $g_{1}\left(z_{1}, z_{2}, z_{3}\right)$ of $(81)$ has the same property. Since the analytic properties of the $f_{1}$ also imply that the $g_{1}$ has corresponding analytic properties, and since the other functions $g_{2}$ and $g_{3}$ can be determined in the same way in which the $g_{1}$ has been determined and will have corresponding properties of continuity and of analyticity, we see that the proof of Lemma 7 is complete.

We may summarize the discussion of this and the preceding sections in the concluding

THEOREM 2. There exists a transformation of the type

$$
x_{i}=x_{i}\left(z_{1}^{*}, z_{2}^{*}, z_{3}^{*}\right) \quad(i=1,2,3),
$$

where the functions $x_{i}\left(z_{1}^{*}, z_{2}^{*}, z_{3}^{*}\right)$ are of class $C^{\infty}$ in lsome neighborhood $\left(z_{1}^{*}, z_{2}^{*}, z_{3}^{*}\right)=(0,0,0)$ and are analytic for $z_{1}^{*} \neq 0, z_{2}^{*} \neq 0, z_{3}^{*} \neq 0$, under which the differential equations (7) are equivalent to the differential equations

$$
\frac{d z_{i}^{*}}{d t}=m_{i} z_{i}^{*} \quad(i=1,2,3),
$$

in a certain neighborhood of the origin, which point remains invariant under the transformation. Furthermore, when.the equations (85) are solved for the $z_{i}^{*}$ as functions of the $x_{j}$ and are expanded as formal power series in the $x_{j}$, if the equations (7) have the form (12) the formal power series for $z_{1}^{*}$ will formally satisfy the partial differential equation (13), and the formal power series for $z_{2}^{*}$ and $z_{3}^{*}$ will formally satisfy the analogues of (13) in $\phi_{2}$ and $\phi_{3}$.

HARVARD UNIVERSTTY,

Cambrimge, Mass. 